\title{
Dynamic BOLD functional connectivity in humans and its electrophysiological correlates
}

\author{
Enzo Tagliazucchi *, Frederic von Wegner, Astrid Morzelewski, Verena Brodbeck and Helmut Laufs
}

Neurology Department and Brain Imaging Center, Goethe University, Frankfurt am Main, Germany

\section{Edited by:}

Johanna Zumer, Radboud University

Nijmegen, Netherlands

Reviewed by:

Alexander Schäfer, Max Planck

Institute for Human Cognitive and

Brain Sciences, Germany

Pablo Barttfeld, Universidad de

Buenos Aires, Argentina

Marieke L. Scholvinck, Ernst

Strungmann Institute for

Neuroscience in Cooperation with

Max Planck Society, Germany

${ }^{*}$ Correspondence:

Enzo Tagliazucchi, Neurology

Department and Brain Imaging

Center, Goethe University, Frankfurt

am Main, Germany.

e-mail:nztglzcch@gmail.com
Neural oscillations subserve many human perceptual and cognitive operations. Accordingly, brain functional connectivity is not static in time, but fluctuates dynamically following the synchronization and desynchronization of neural populations. This dynamic functional connectivity has recently been demonstrated in spontaneous fluctuations of the Blood Oxygen Level-Dependent (BOLD) signal, measured with functional Magnetic Resonance Imaging (fMRI). We analyzed temporal fluctuations in BOLD connectivity and their electrophysiological correlates, by means of long $(\approx 50 \mathrm{~min})$ joint electroencephalographic (EEG) and fMRI recordings obtained from two populations: 15 awake subjects and 13 subjects undergoing vigilance transitions. We identified positive and negative correlations between EEG spectral power (extracted from electrodes covering different scalp regions) and fMRI BOLD connectivity in a network of 90 cortical and subcortical regions (with millimeter spatial resolution). In particular, increased alpha $(8-12 \mathrm{~Hz})$ and beta $(15-30 \mathrm{~Hz})$ power were related to decreased functional connectivity, whereas gamma $(30-60 \mathrm{~Hz})$ power correlated positively with BOLD connectivity between specific brain regions. These patterns were altered for subjects undergoing vigilance changes, with slower oscillations being correlated with functional connectivity increases. Dynamic BOLD functional connectivity was reflected in the fluctuations of graph theoretical indices of network structure, with changes in frontal and central alpha power correlating with average path length. Our results strongly suggest that fluctuations of BOLD functional connectivity have a neurophysiological origin. Positive correlations with gamma can be interpreted as facilitating increased BOLD connectivity needed to integrate brain regions for cognitive performance. Negative correlations with alpha suggest a temporary functional weakening of local and long-range connectivity, associated with an idling state.

Keywords: dynamic connectivity, EEG-fMRI, resting state, brain networks, brain oscillations

\section{INTRODUCTION}

Neural oscillations at specific frequency bands reflect a wide repertoire of brain states, ranging from active cognitive performance to idling rest, sleep and other states of diminished awareness (Buzsáki, 2006). Experimental results relate power increases and synchronization in the gamma frequency band $(\approx 40 \mathrm{~Hz})$ to the performance of perceptual and cognitive operations, including attention (Womelsdorf and Fries, 2007), conscious perception (Melloni et al., 2007), and decision making (Donner et al., 2009). In particular, the ubiquity of gamma band synchronization in human brain function has led to its postulation as a fundamental process subserving an elementary operation of cortical computation (Fries, 2009). When compared to other alternatives, like rate encoding, neural integration by temporal synchronization offers many theoretical advantages, such as, a faster temporal realization and no ambiguities due to modulation of firing rate by other causes (e.g., receptive field tuning or other concurrently integrated neural groups) (Singer et al., 2010). On the other hand, increased power in slower frequencies has been linked with an idling state of the brain (Pfurtscheller et al., 1996) and with functional inhibition of task-irrelevant regions, facilitating routing of information to task-relevant regions (Klimesch et al., 2007; Jensen and Mazaheri, 2010). Slower frequencies, such as delta $(<4 \mathrm{~Hz})$, arise during sleep and are hypothesized to reflect diminished temporal complexity underlying loss of conscious awareness (Tononi et al., 1994; Tononi, 2004, 2008).

This integration and inhibition of cortical processes through local and long-range neural synchrony translates into temporal fluctuations of functional connectivity, observable by electrophysiological recordings (such as scalp electroencephalographyEEG). Dynamic functional connectivity has only recently been demonstrated for Blood Oxygen Level-Dependent (BOLD) signals, measured with functional Magnetic Resonance Imaging (fMRI) (Chang and Glover, 2010; Tagliazucchi et al., 2010a,b, 2012; Handwerker et al., 2012; Hutchison et al., 2012; Petridou et al., 2012; Smith et al., 2012). Given the precise spatial resolution and whole brain coverage allowed by fMRI, understanding the relationship between brain rhythms and BOLD connectivity could contribute to the identification of the functional roles of each oscillation at rest and during a task. A necessary first step in this direction is to understand the relationship between dynamic BOLD connectivity fluctuations and changes in the local 
synchronization of cortical neural populations, as indexed by frequency-specific EEG power. Large efforts have been devoted to the identification of the electrophysiological counterparts of fMRI BOLD signal amplitude during wakeful rest. Along this line, it has been demonstrated that EEG power in the alpha band correlates negatively with spontaneous BOLD amplitude fluctuations in lateral frontal and parietal cortices, whereas power in the beta band correlates positively with regions comprising the Default Mode Network [a network of task de-activated regions (Raichle et al., 2001; Laufs et al., 2003a,b)]. Further evidence for a specific profile of EEG spectral power associated with different Resting State Networks (RSN) was provided in Mantini et al. (2007). In monkey studies, Local Field Potentials (LFP) exhibit widespread positive correlations with BOLD fMRI, mainly in the gamma frequency band (Schölvinck et al., 2011). These reports during rest are complemented by studies mapping the electrophysiological correlates of the BOLD signal during task performance and stimulation, also demonstrating that LFP gamma frequency is a main contributor to the hemodynamic signal (Logothetis et al., 2001; Logothetis and Pfeuffer, 2004). In spite of this large body of studies relating rhythmic neural activity to BOLD signal amplitude, a relationship between BOLD functional connectivity and the power of band-specific scalp oscillations remains a relatively unexplored possibility.

In this paper we study such possibility by correlating dynamic BOLD connectivity fluctuations with changes in EEG power in a group of awake human subjects, as well as in a group of subjects undergoing vigilance transitions between wakefulness and light sleep, whose EEG recordings exhibit marked changes in spectral power over time. The inclusion of these subjects can be seen as a (physiological) manipulation in one of the correlated variables (EEG power in different bands) to test the effect on the other (BOLD functional connectivity). We hypothesize that band-specific electrophysiological spectral changes will correlate with fluctuations in BOLD connectivity and that increased power in fast EEG frequency bands (such as gamma) will correlate with increased BOLD connectivity, considering the ubiquity of gamma oscillations in long-range neural synchronization. On the other hand, activity in the alpha and peri-central (or rolandic) beta bands will not be expected as a contributor to increased BOLD connectivity, given their hypothesized relationship with idling and functional inhibition. We also hypothesize that changes in functional connectivity over time will impact on graph theoretical metrics, which are established descriptors of the global connectivity network architecture. The study of global brain connectivity patterns has been, in recent years, greatly aided by the introduction of graph theoretical concepts (Sporns et al., 2004; Bullmore and Sporns, 2009). These allow to extract information from a network representation of brain functional connectivity, in which nodes represent distinct brain regions and links represent synchronized activity between those regions. The use of these methods not only allows the evaluation of changes in functional connectivity strength, but also in the topological reorganization of brain connectivity and its interpretation in terms of efficient information processing (Bullmore and Sporns, 2012). Finally, an important consequence of our study will be to confirm a neurobiological origin for BOLD connectivity fluctuations, given concerns that they might arise due to motion or physiological confounds (Hutchison et al., 2012) and as artifacts due to sliding window correlation analyses (Handwerker et al., 2012).

\section{MATERIALS AND METHODS}

\subsection{EEG-fMRI ACOUISITION AND ARTIFACT CORRECTION}

EEG via a cap (modified BrainCapMR, Easycap, Herrsching, Germany) was recorded continuously during fMRI acquisition, yielding 1505 volumes of T2*-weighted echo planar images with $\mathrm{TR} / \mathrm{TE}=2080 / 30 \mathrm{~ms}$, matrix $64 \times 64$, voxel size $3 \times 3 \times 2 \mathrm{~mm}^{3}$, distance factor $50 \%$ and FOV $192 \mathrm{~mm}^{2}$. Scanning was performed at $3 \mathrm{~T}$ (Siemens Trio, Erlangen, Germany) together with an optimized polysomnographic setting including chin and tibial EMG, ECG, EOG recorded bipolarly (sampling rate $5 \mathrm{kHz}$, low pass filter $1 \mathrm{kHz}$ ), and $30 \mathrm{EEG}$ channels recorded with $\mathrm{FCz}$ as the reference (sampling rate $5 \mathrm{kHz}$, low pass filter $250 \mathrm{~Hz}$ ). Pulse oxymetry and respiration were recorded via sensors from the Trio (sampling rate $50 \mathrm{~Hz}$ ) and MR scanner compatible devices (BrainAmp MR+, BrainAmp ExG; Brain Products, Gilching, Germany).

MRI and pulse artifact correction were performed based on the average artifact subtraction (AAS) method (Allen et al., 1998) as implemented in Vision Analyzer2 (Brain Products, Germany) followed by objective (CBC parameters, Vision Analyzer) ICAbased rejection of residual artifact-laden components after AAS, resulting in EEG with a sampling rate of $250 \mathrm{~Hz}$. EEG was rereferenced to common average. Sleep stages were scored manually by an expert according to the AASM criteria (AASM, 2007).

\subsection{SUBJECTS AND DATASETS}

A total of 15 awake subjects were included in the study (10 female, age $26.2 \pm 6$ ), together with an independent group of 13 subjects undergoing vigilance transitions between wakefulness and light sleep or N1 sleep ( 8 female, age $23.3 \pm 3.4$ ). In all cases, written informed consent and approval by the local ethics committee were obtained. Both groups were extracted from a larger dataset with the following inclusion criteria: for the first group, subjects did not show any period of sleep (as determined by AASM sleep scoring criteria). For the second group, subjects showed only epochs of wakefulness and at least $20 \%$ of light (N1) sleep (the total time spent in N1 sleep was $13.09 \pm 6.26 \mathrm{~min}$ ). According to AASM criteria, an epoch of $\mathrm{N} 1$ sleep was scored whenever alpha waves were replaced by lowamplitude and lower frequency $(4-7 \mathrm{~Hz})$ waves occupying $>50 \%$ of the epoch. In the cases in which alpha waves were not visible during wakefulness, the presence of 4-7 Hz oscillations with slowing of background activity (compared with wakefulness), vertex sharp waves, or slow eye movements were also used to score N1 sleep.

\section{3. fMRI PRE-PROCESSING}

Using Statistical Parametric Mapping (SPM 8, http://www.fil.ion. ucl.ac.uk/spm) EPI data were realigned, normalized (MNI space), and spatially smoothed (Gaussian kernel, $8 \mathrm{~mm}^{3}$ full width at half maximum). Cardiac-, respiratory- [both estimated with the RETROICOR method (Glover et al., 2000)] and motion-induced noise were regressed out. fMRI data was bandpass filtered in the range $0.01-0.1 \mathrm{~Hz}$ using a 6th order Butterworth filter. 


\subsection{TIME DEPENDENT CORRELATION MATRIX}

To study the covariance of functional connectivity and EEG power in different frequency bands, an estimate of how the former changes over time is necessary. For this purpose (following, Hutchison et al., 2012; Fraiman and Chialvo, 2012) a sliding window analysis was employed, with a window length of $\approx 2 \mathrm{~min}$ ( 60 volumes). This window length was chosen because it is relatively short compared to the length of the experiment $(\approx 50 \mathrm{~min})$, while allowing good functional connectivity estimates (Van Dijk, 2010). As a first step, the average BOLD signal was extracted from each one of the 90 cortical and subcortical regions defined by the AAL template (Tzourio-Mazoyer et al., 2002) (information on all the regions is provided in Table $\mathbf{1}$ ). These time series are notated as $x_{i}, 1 \leq i \leq 90$. Next, the time-dependent correlation matrix was obtained as follows:

$$
\begin{aligned}
C_{i j}(t)=\frac{\sum_{n=t}^{t+k}\left(x_{i}(n)-\frac{1}{k} \sum_{m=t}^{t+k} x_{i}(m)\right) \times}{\sqrt{\sum_{n=t}^{t+k}\left(x_{j}(n)-\frac{1}{k} \sum_{m=t}^{t+k} x_{j}(m)\right)}} \\
\sqrt{\sum_{n=t}^{t+k}\left(x_{j}(n)-\frac{1}{k} \sum_{m=t}^{t+k} x_{i}^{t+k}(m)\right)^{2} \times} \times \\
=\frac{\left\langle\left(x_{i}(t: t+k)-\left\langle x_{i}(t: t+k)\right\rangle\right) \times\right.}{\left.\sigma\left(x_{j}(t: t+k)-\left\langle x_{j}(t: t+k)\right\rangle\right)\right\rangle}
\end{aligned}
$$

The second expression was simplified using a notation similar to MATLAB vector syntax, in which $x(n: m)$ represents the portion of $x$ ranging from the $n$-th to the $m$-th entries (i.e., the portion of the time series $x$ ranging from the $n$-th to the $m$-th measurement). Thus, $C_{i j}(t)$ is the linear correlation between $x_{i}$ and $x_{j}$ during a window of length $k$ starting from $t$. As mentioned above, $C_{i j}(t)$ was computed with $k=60$, which corresponds to $\approx 2$ min.

\subsection{EEG POWER, MOTION, CARDIAC, AND RESPIRATORY TIME COURSES}

Next, time courses for the variables to be correlated with BOLD connectivity were obtained. For this purpose, a sliding window average was applied, with the same window length $(\approx 2 \mathrm{~min})$ used to construct the time-dependent functional connectivity matrix $\left(C_{i j}(t)\right)$. Given a time series $y$, the computation is as follows:

$$
Y(t)=\frac{1}{k} \sum_{n=t}^{t+k} y(n)=\langle y(t: t+k)\rangle
$$

which gives the desired result when $k=60$. These sliding window-averaged time courses were obtained for delta $(0.4-4 \mathrm{~Hz})$, theta $(4-8 \mathrm{~Hz})$, alpha $(8-12 \mathrm{~Hz})$, sigma $(12-15 \mathrm{~Hz})$, beta $(15-30 \mathrm{~Hz})$, and gamma $(30-60 \mathrm{~Hz})$ power, averaged over frontal (channels F1, Fz, and F2), central (channels $\mathrm{C} 1, \mathrm{Cz}$, and $\mathrm{C} 2$ ), and occipital (channels $\mathrm{O} 1, \mathrm{Oz}$, and O2) EEG and also for the cardiac and respiratory time series estimated with the RETROICOR method. The sliding window-averaged time course for the relative displacement with respect to an arbitrary volume was also obtained, computed as $D=\sqrt{D_{x}^{2}+D_{y}^{2}+D_{z}^{2}}$, where $D_{x}$, $D_{y}$, and $D_{z}$ are the estimated displacements (after spatial realignment with SPM8) in the $x, y$, and $z$ axis, respectively. For an overview of the data analysis, see Figure 1.

\subsection{CORRELATION BETWEEN FMRI CONNECTIVITY FLUCTUATIONS AND ELECTROPHYSIOLOGICAL TIME SERIES}

Next, the time-dependent BOLD functional connectivity between each pair of regions [as represented in $\left.C_{i j}(t)\right]$ was correlated with the sliding window-averaged time series of electrophysiological origin. Even though cardiac, respiratory and motion time series were regressed out of the signal at the pre-processing stage, they were still kept as partial regressors in the analysis. Therefore, we obtained the partial correlation between $C_{i j}(t)$ for $1 \leq i \leq 90$, $1 \leq j \leq i-1$ and the different frequency bands from frontal, central and occipital channels:

$$
R_{C}(X, Y)=\min _{Z} R(X, Y \mid Z)
$$

Here, $Z$ runs through all the other variables involved in the partial correlation. $R(X, Y \mid Z)$ is defined as follows,

$$
R(X, Y \mid Z)=\frac{R(X, Y)-R(Y, Z)}{\sqrt{1-R(X, Z)^{2}} \sqrt{1-R(Y, Z)^{2}}}
$$

in which $R(X, Y)$ is the linear correlation between both variables, as in Equation (1).

\subsection{TIME-DEPENDENT GRAPH METRICS}

Then we analyzed the presence of correlations between the time development of common graph metrics associated with the global fMRI functional connectivity networks and time courses of EEG power fluctuations in different frequency bands. Graph metrics summarize topological information (i.e., not explicitly related to brain anatomy or geometry) about brain connectivity. For this purpose, a graph representation of functional connectivity is needed, in which each node represents a brain anatomical region and a link between two nodes represents significant functional connectivity between the BOLD signals from the nodes. The study of these metrics allows to map changes in global connectivity architecture [important to understand the information processing capacities of the system (Bullmore and Sporns, 2012)] related to a re-organization of the strongest connections. To obtain this representation, the correlation matrix $C_{i j}(t)$ was thresholded to obtain the time-dependent adjacency matrix (Bassett et al., 2010) $A_{i j}(t)$ as follows:

$$
A_{i j}(t)= \begin{cases}0 & \text { if } C_{i j}(t)<\rho \\ 1 & \text { if } C_{i j}(t) \geq \rho\end{cases}
$$

In the adjacency matrix $A_{i j}(t)$ a 1 represents a link between nodes $i$ and $j$ at time $t$. The arbitrary parameter $\rho$ was chosen so that in all cases the resulting networks had a link density of 0.10 , i.e., $10 \%$ of the total number of possible links in the networks were actually present. For each time step, a number of graph metrics using the MATLAB Brain Connectivity Toolbox (Rubinov 
Table 1 | Region number, name, abbreviation, system membership (from Achard et al., 2006), and anatomical coordinates (for the center of mass of the 90 cortical and subcortical regions defined in the AAL template).

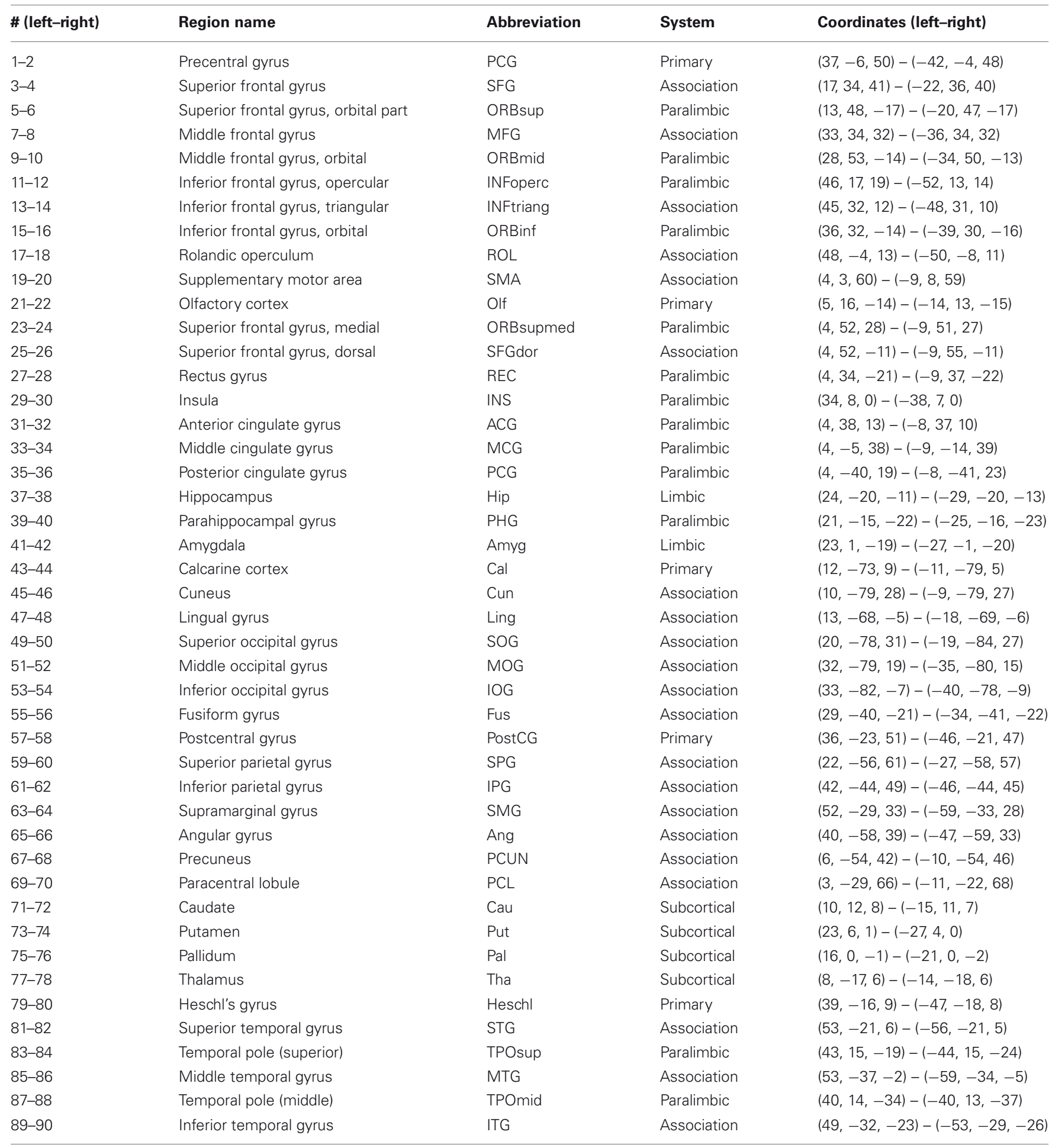

and Sporns, 2010) was computed. Heuristic definitions are provided below [illustrations exemplifying the different metrics can be found in Figure 10A, for a detailed review see Sporns et al. (2004), Bullmore and Sporns (2009), and Rubinov and Sporns (2010)]:
- Clustering coefficient $(\gamma)$. The clustering coefficient of a given node is the probability that two nodes which are connected to it, are connected to one another. The clustering coefficient is then computed as the average of the clustering coefficient of all individual nodes. 

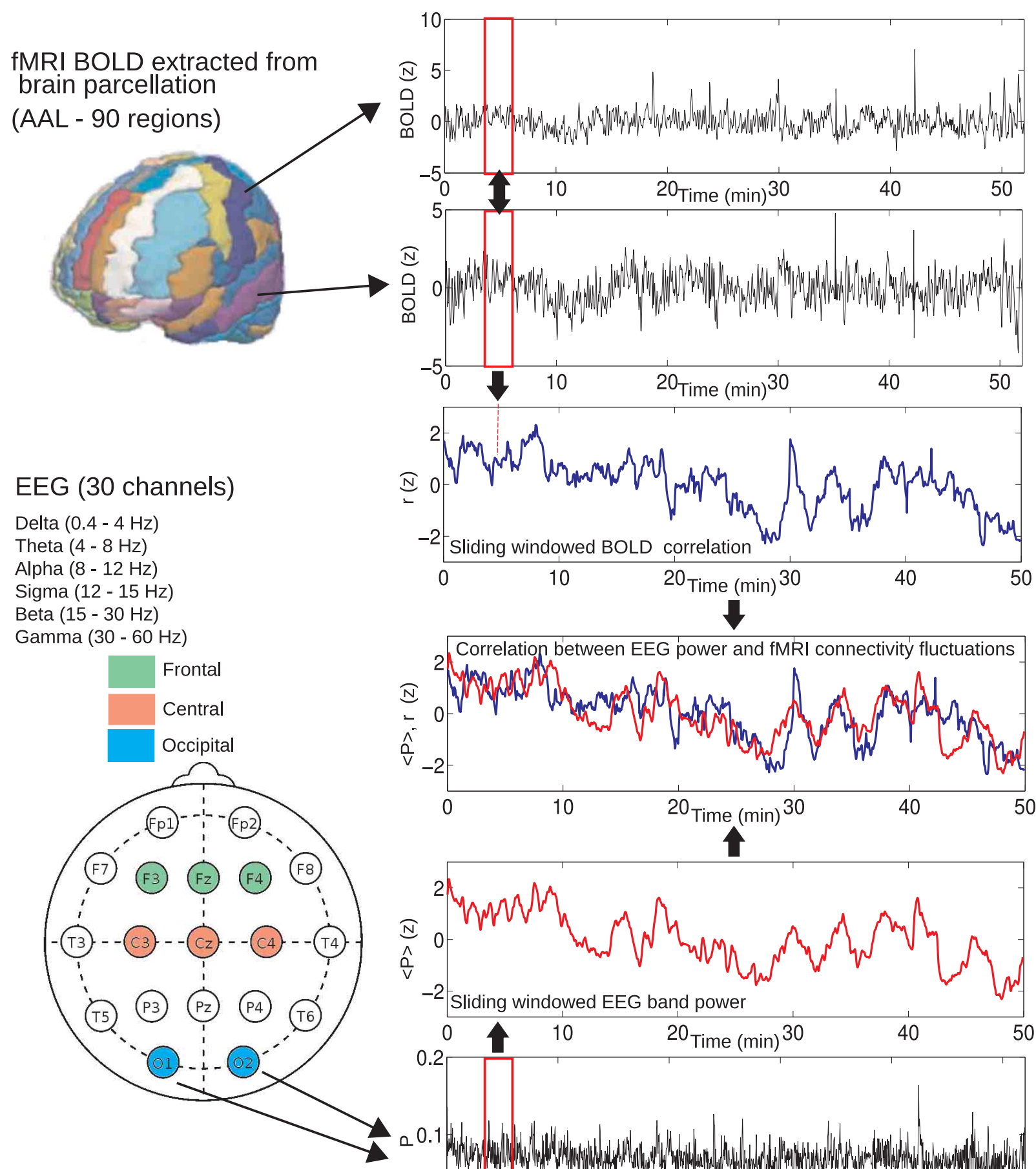

FIGURE 1 | Method used to compute BOLD connectivity fluctuations and correlations with EEG power fluctuations. For each pair of regions, average BOLD signals were extracted and correlated using a sliding window of 60 volumes $(\approx 2 \mathrm{~min})$. This resulted in a connectivity estimate over time. A similar sliding window approach was used to obtain the average EEG power from different frequency bands (delta, theta, alpha, sigma, beta, and gamma), averaged from different locations (frontal, central, and occipital). As a final step, these EEG power fluctuations were correlated with BOLD connectivity for each pair of regions and correlations were tested for statistical significance (Student's $t$-test, FDR controlled for multiple comparisons). 
- Average path length $(\lambda)$. The distance between node $i$ and node $j$ is the minimum number of links which have to be crossed when going from $i$ to $j$. The average path length is the average of the distance between all possible pairs of nodes in the network.

- Betweeness ( $\beta$ ). A path between node $i$ and node $j$ is defined as the sequence of linked nodes which have to be visited to go from $i$ to $j$. A minimum path between node $i$ and node $j$ is a path with a number of links equal to the distance between $i$ and $j$. The betweeness of a given node in the network is defined as the number of minimum paths of which that node is a member. The betweeness of the network is computed as the average betweeness of all individual nodes.

- Small-worldness $(\sigma)$. To compute the small-worldness coefficient, networks are first randomized, scrambling their links at random with the constraint of a preserved connectivity distribution. Then, the clustering coefficient $\left(\gamma_{\text {Rand }}\right)$ and the average path length $\left(\lambda_{\text {Rand }}\right)$ of the randomized networks are computed. The small-worldness coefficient is then obtained as $\sigma=\frac{\gamma^{*}}{\lambda^{*}}$, where $\gamma^{*}=\frac{\gamma}{\gamma_{\text {Rand }}}$ and $\lambda^{*}=\frac{\lambda}{\lambda_{\text {Rand }}}$. A value of $\sigma>1$ is regarded as indicator of small-world structure in the network (Humphries et al., 2006).

After obtaining the time courses of $\gamma(t), \lambda(t), \beta(t)$, and $\sigma(t)$, the presence of correlations with time courses of EEG power in the delta, theta, alpha, sigma, beta, and gamma bands was studied (taking cardiac, respiratory, and motion time series into account as partial regressors).

\subsection{STATISTICAL TESTING}

To test for statistical significance, all correlation values were first transformed to z-scores using the Fisher transform, given by $z=\operatorname{artanh}(r)$. An ANOVA test was used to study the effect of EEG frequency band on the correlation coefficients with BOLD connectivity time courses. Then, Student's $t$-tests were performed with the null hypothesis of zero correlation. To correct for the multiple comparisons performed the False Discovery Rate (FDR) method was used, with $q=0.05$. For the correlation between graph metrics and EEG power time courses Bonferroni correction was applied with $n=6$ (frequency bands) $\times 3$ (number of channel groups $) \times 4$ (number of graph metrics $)=72$.

\section{RESULTS}

\subsection{DYNAMIC SPONTANEOUS BOLD CONNECTIVITY FLUCTUATIONS}

We started by assessing the presence of temporal fluctuations in BOLD connectivity, a necessary first step to perform the correlation analysis with EEG power fluctuations.

Functional connectivity between brain regions fluctuated widely over time, consistently with previous reports (Chang and Glover, 2010; Handwerker et al., 2012; Hutchison et al., 2012). An example of this dynamic functional connectivity is shown in Figure 2A, in which the complete functional connectivity matrix of a single subject is presented in intervals of 2 min. It is clear, by simple visual inspection, that the connectivity matrix fluctuates over time, with periods of overall decreased connectivity alternating with periods of globally increased connectivity [termed hypersynchrony in a previous study (Hutchison et al., 2012)]. As another example, in Figure 2B the time courses of connectivity between left and right thalamus are shown for the same subject and also for a subject undergoing vigilance transitions between wakefulness and light (N1) sleep. Correlation between the homeotopic BOLD signals clearly changes over time, alternating between a correlation close to the highest possible value $(r=1)$ and a complete discoordination $(r=0)$, in spite of strong average inter-thalamic connectivity $(r>0.5)$. The extent of functional connectivity variation was quantified by taking the standard deviation (SD) of the connectivity time course between all pairs of regions. The average $\mathrm{SD}$ for both groups are shown in Figure 2C, together with their difference. "Blocks" of lower SD can be observed grouped around the diagonal of the matrix, indicating groups of (anatomically) neighboring regions with lower functional connectivity variability over time (for example, occipital regions, ranging from regions \#43-44 to \#55-56), while displaying a higher variability in their connections with the rest of the brain (off-diagonal entries). While there were no significant differences surviving multiple comparison correction, there was a trend of higher SD in frontal connectivity for the group of awake subjects and of higher SD in cortico-thalamic connectivity for the group of subjects exhibiting vigilance transitions between wakefulness and light sleep.

\subsection{CORRELATIONS WITH SPONTANEOUS EEG POWER FLUCTUATIONS}

Next, we studied the correlations between EEG power and BOLD connectivity time courses, in order to identify EEG frequency bands involved in connectivity changes over time. We first studied the effect of the different EEG frequency bands on the correlation coefficient with dynamic BOLD connectivity. Results for both groups are presented in Figure 3, in which a frequency-dependent effect can be readily appreciated. In what follows, we explore these correlations specific to each frequency band, topographic location, and group of subjects.

Results for the group of awake subjects are presented in Figure 4. Widespread negative correlations between BOLD connectivity fluctuations and central alpha and beta power were detected. Positive correlations were found with central, frontal, and occipital gamma power fluctuations, with correlations with central channels being more widespread than the others.

Correlations for the group of subjects undergoing vigilance changes are presented in Figure 5. Positive correlations were found with central delta power fluctuations, with a spatial emphasis in frontal regions and between frontal and temporal regions. Negative correlations with frontal and occipital alpha also affected predominantly frontal BOLD connectivity, whereas the central sigma band showed more distributed negative correlations. Finally, very few positive correlations with the frontal gamma time course were found.

We also directly tested whether functional connectivity changes correlated with cardiac, respiratory, and motion time series. Negative results were found for cardiac and motion time series, and only spurious positive correlations for the group of awake subjects (four pairs of regions) and for the subjects with fluctuating vigilance (two pairs of regions). 
A
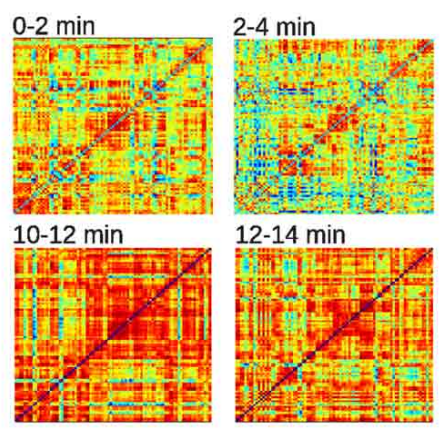
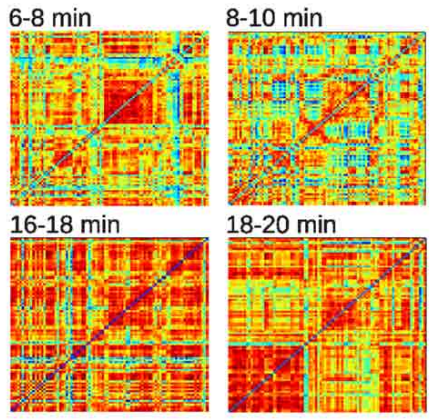

B
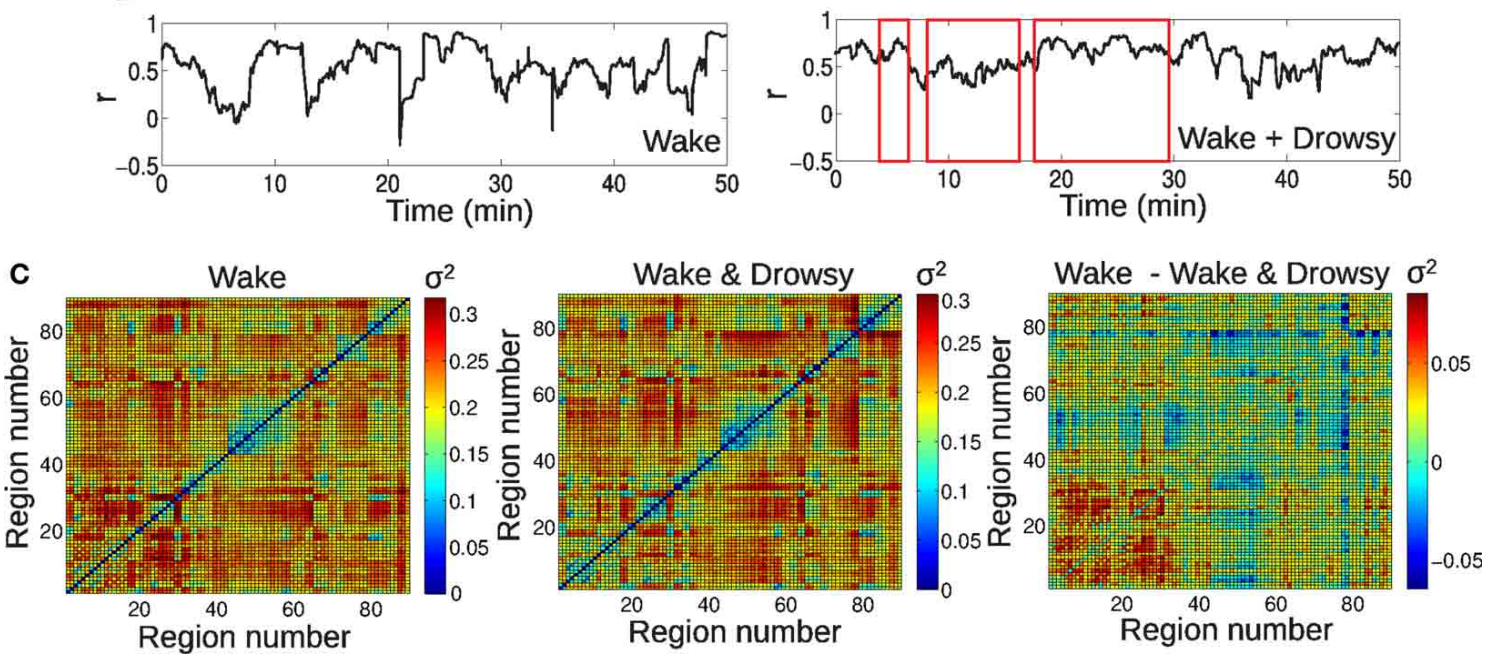

FIGURE 2 | Large-scale spontaneous BOLD functional connectivity fluctuations. (A) BOLD correlation matrices for a single subject, in intervals of $2 \mathrm{~min}$. (B) Time series of inter-hemispheric thalamic connectivity for an awake subject and a subject undergoing vigilance transitions between wakefulness and light sleep (periods of light sleep are marked by red rectangles). (C) Standard deviation of BOLD connectivity time series for each pair of regions, for both groups (wakefulness and wakefulness \& light sleep) and their difference. For the AAL regions associated with region numbers, see Table 1.

\subsection{NODES WITH CONNECTIVITIES MOST INFLUENCED BY EEG POWER FLUCTUATIONS}

To extract information about the network of connections correlated with EEG power in specific frequency bands, we computed for each node the number of connections with other nodes which were affected by spontaneous power fluctuations. This is equivalent to the degree (D) of each region or node, if one defines a network whose links are the EEG power-modulated connections.

After having obtained the degree for each region, areas were ranked in decreasing order. Results are shown in Figure 6 for the group of awake subjects and Figure 7 for the group of subjects undergoing vigilance transitions. In the insets, regions corresponding to the 4 th quintile of the distributions are displayed overlaid on a standard MNI T1 template (lighter colors represent a higher number of correlations). For the group of awake subjects, the region with the highest number of negative correlations with central alpha was the thalamus. Other top-ranked regions were sub-cortical and bilateral frontal. For correlations with beta, highest ranked regions were also subcortical (pallidum, putamen and caudate nucleus). Top-ranked regions with positive functional connectivity correlations in the central gamma band were mostly frontal. The same was observed for correlations with occipital gamma (with the inclusion of the bilateral insular cortices as the regions with the highest degree). In correlations with frontal gamma, on the other hand, frontal regions were second to precuneus, temporal and parietal areas (Figure 6).

For the group of subjects undergoing vigilance transitions, superior and middle frontal gyri and cingulate regions exhibited the largest number of positive correlations with delta power. The same was observed for negative correlations with frontal and occipital alpha (including also insular and precuneal cortices) and central sigma (in all three cases, the top-ranked region was the dorsal part of the superior frontal gyrus). The top-ranked region for positive correlations with frontal gamma was the gyrus rectus followed by occipital regions.

\subsection{EEG POWER FLUCTUATIONS AND CONNECTIVITY BETWEEN DIFFERENT SETS OF BRAIN REGIONS}

We quantified the number of connections modulated by EEG power between different sets of brain regions, in order to reveal 


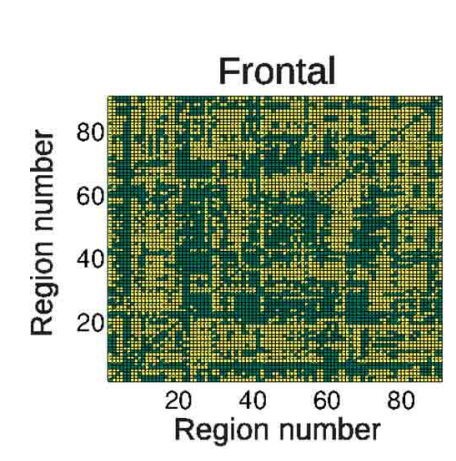

\section{Effect of EEG band (Wake)}
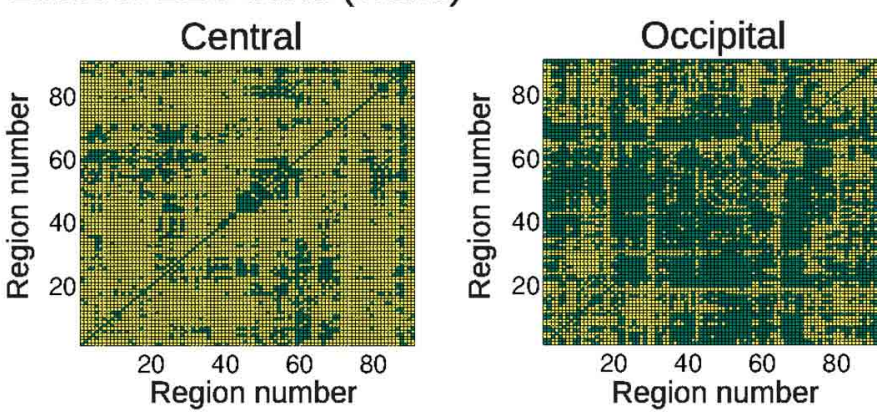

Effect of EEG band (Wake \& Drowsy)
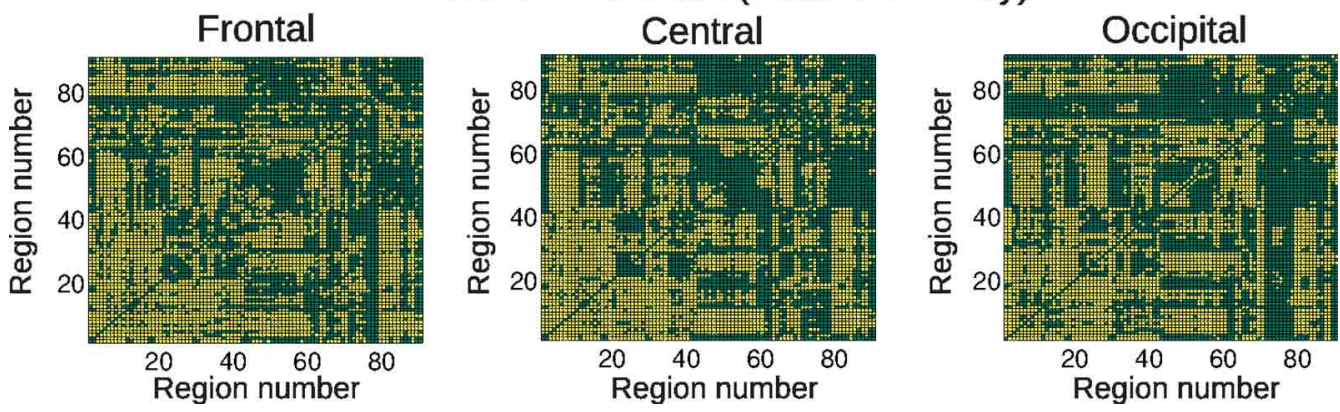

FIGURE 3 | Connections showing a significant (colored in yellow) effect of EEG frequency band in the correlation coefficient between BOLD functional connectivity and spontaneous EEG power fluctuations, for the three scalp regions defined in Figure 1 (frontal, central, and occipital) and both groups (wakefulness and wakefulness \& light sleep). For the AAL regions associated with region numbers, see Table $\mathbf{1 .}$ frequency-specific changes in communication between brain systems.

For that purpose, a previously introduced classification of each region into five categories was followed, comprising primary sensory, association, subcortical, limbic, and paralimbic areas (Achard et al., 2006) (the system membership of AAL regions can be found in Table 1). The network traffic between each pair of systems was computed as the normalized number of connections which covary negatively or positively with spontaneous EEG power fluctuations. Results (normalized by the total number of possible connections between each pair of systems) are shown in Figure 8 for the group of awake subjects and in Figure 9 for the group of subjects undergoing wakefulness-light sleep transitions. In the first group, EEG alpha power mediated decreased BOLD connectivity mostly between subcortical areas, association and primary cortices. The effect of central beta was marked by changes affecting almost solely connections between subcortical and association areas. Positive correlations with central gamma were more widespread but again peaking at the interaction between subcortical and association systems. A similar effect was observed for correlations with occipital gamma (affecting connections between association areas and all other systems). For frontal gamma power, however, we observed increased BOLD connectivity inside (and not between) primary, subcortical and association areas. For the second group (wakefulness and light sleep) the most salient feature was an involvement of paralimbic connectivity between primary and association cortices. Also, connectivity inside the association system and with the primary cortex was affected for all frequency bands considered.

\subsection{CORRELATION BETWEEN EEG POWER AND SPONTANEOUS GRAPH METRIC FLUCTUATIONS}

The observed correlations between EEG power fluctuations and BOLD connectivity suggest that graph theoretical measures of network organization should also covary with EEG power changes. We studied this possibility for the common graph metrics, clustering coefficient, average path length, betweeness, and small-worldness.

In Figure 10A (left) the definitions provided in the "Materials and Methods" section are illustrated and an example of how the different graph metrics change during the duration of a single subject measurement (Figure 10A, center) is provided, together with histograms of graph metric values for all subjects (Figure 10A, right). Note that while small-worldness $(\sigma)$ is usually greater than 1 [the limit value at which networks are usually considered to be small-world (Humphries et al., 2006; van den Heuvel et al., 2008)], at certain times this value goes below this threshold, highlighting the fact that the claim of small-worldness of brain functional networks originates from average connectivity, but that in fact small-worldness indeed is not given at all times. It must be noted that the use of Pearson correlation to compute functional connectivity networks will result in an overestimation of the number of triangles (i.e., the clustering coefficient) and thus also of the small-worldness (for a discussion see Smith et al., 2011). An overestimation of this index, however, is likely to leave relative differences unaffected, preserving the dynamics. In Figure 10B, the temporal correlations between fluctuations in graph metrics and occipital, frontal and central EEG alpha power are presented (the other frequency bands did 


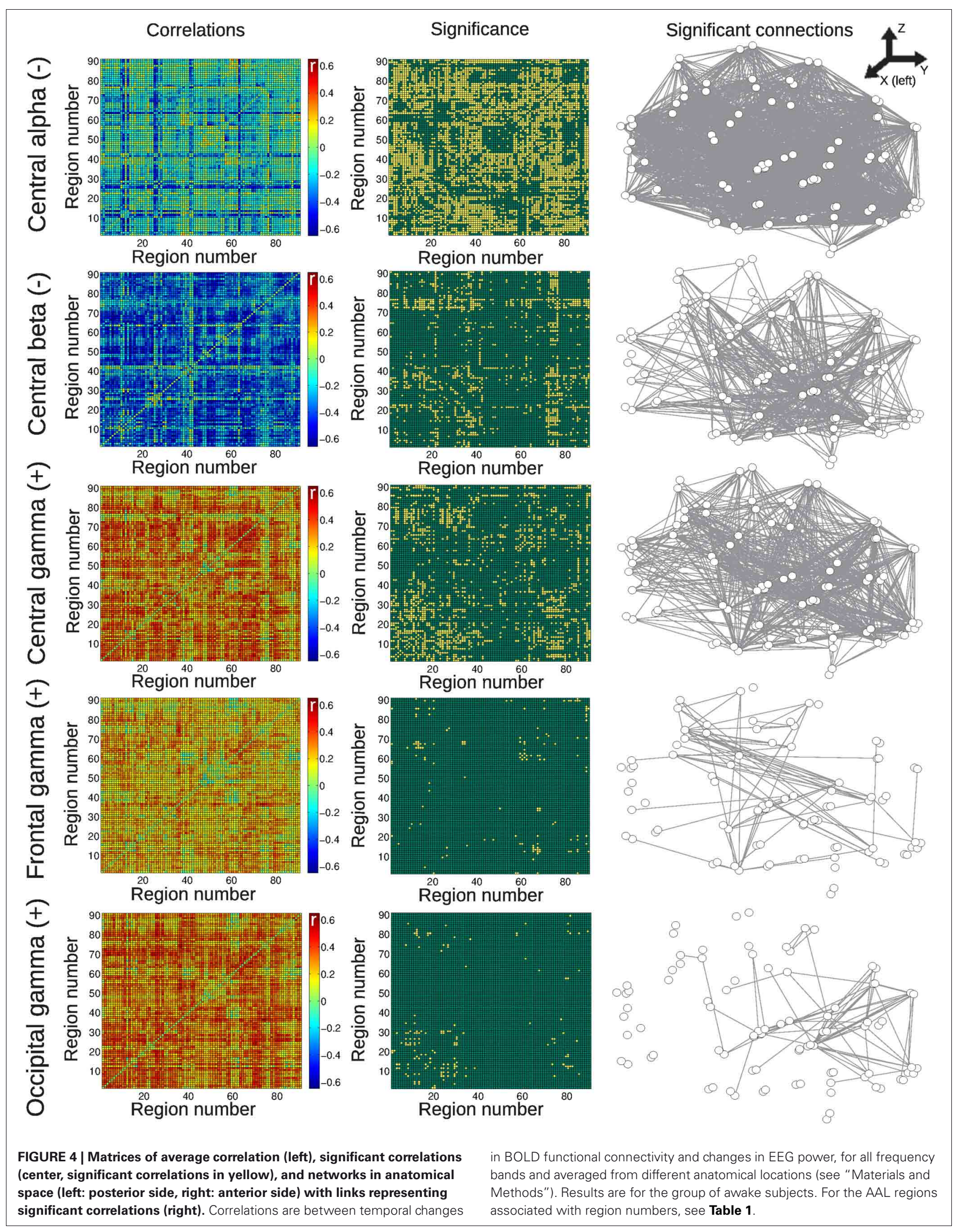




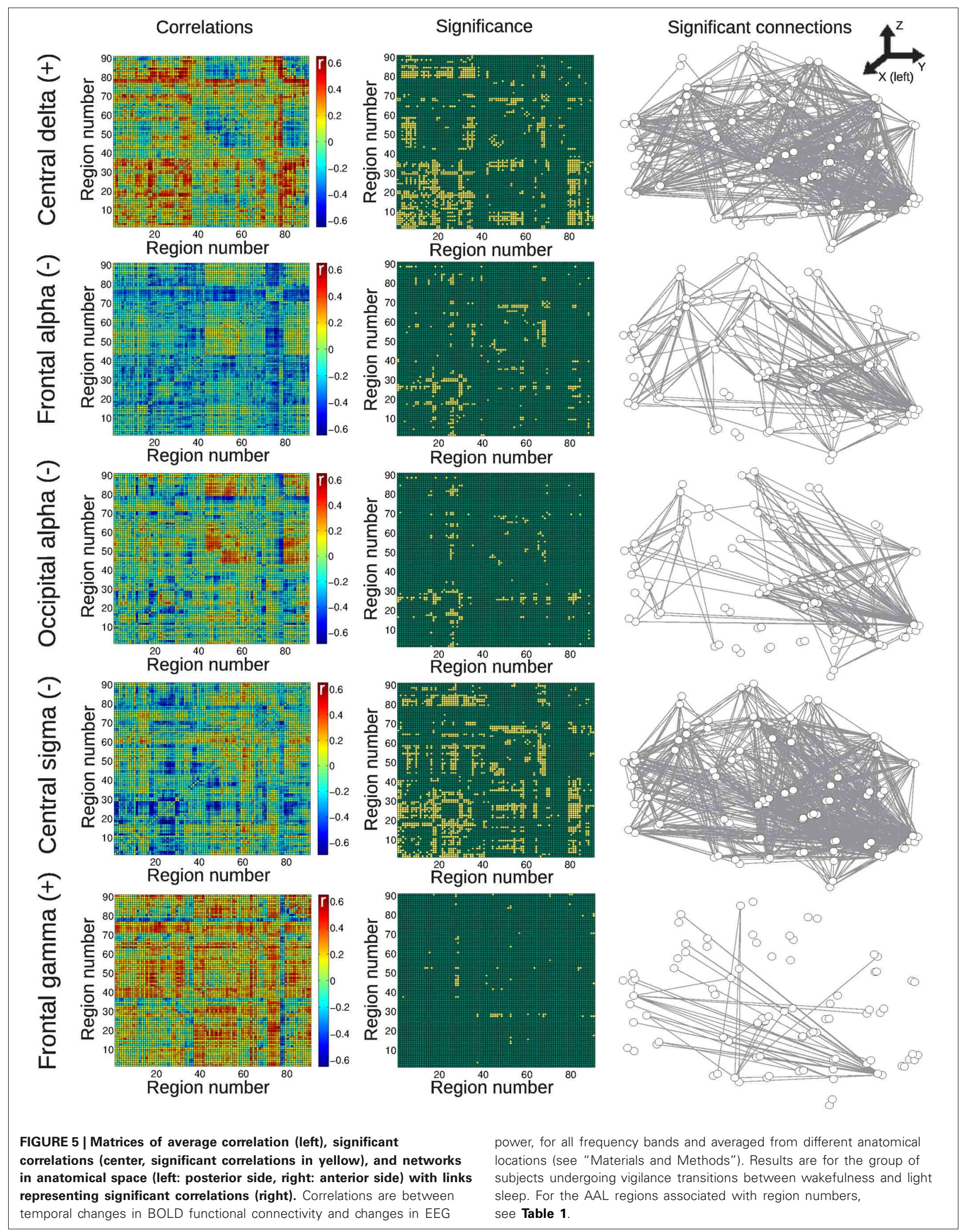



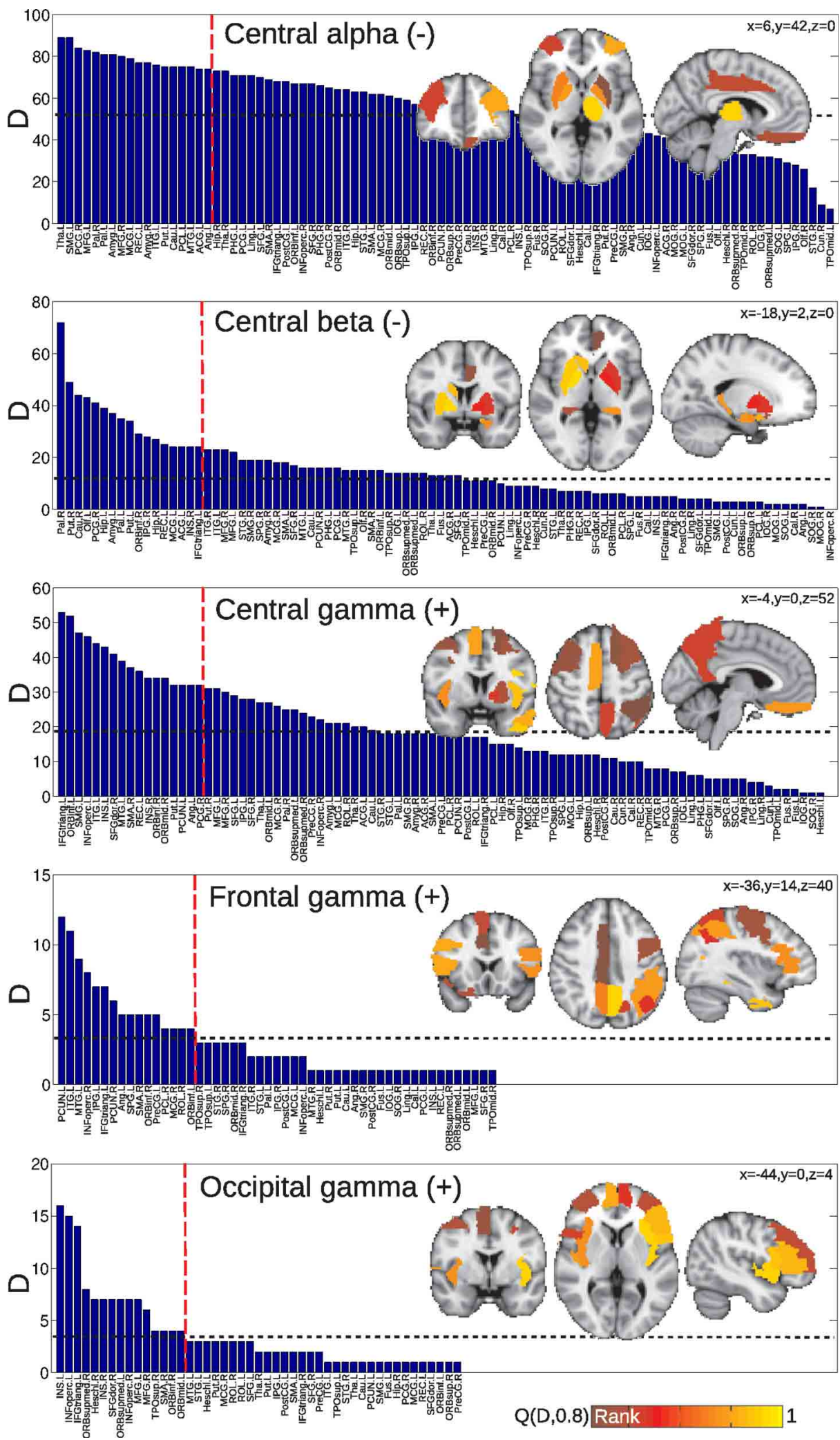

FIGURE 6 | Anatomical regions (or nodes) ranked according to the number of connections attached to them which correlate with EEG power fluctuations in different frequencies. In the inset, the regions corresponding to the top quintile of the distribution-denoted as $Q(D, 0.8)$-are displayed overlaid on a standard MNI T1 template. The horizontal dashed line indicates the mean of the distribution. Results are for the group of awake subjects. 

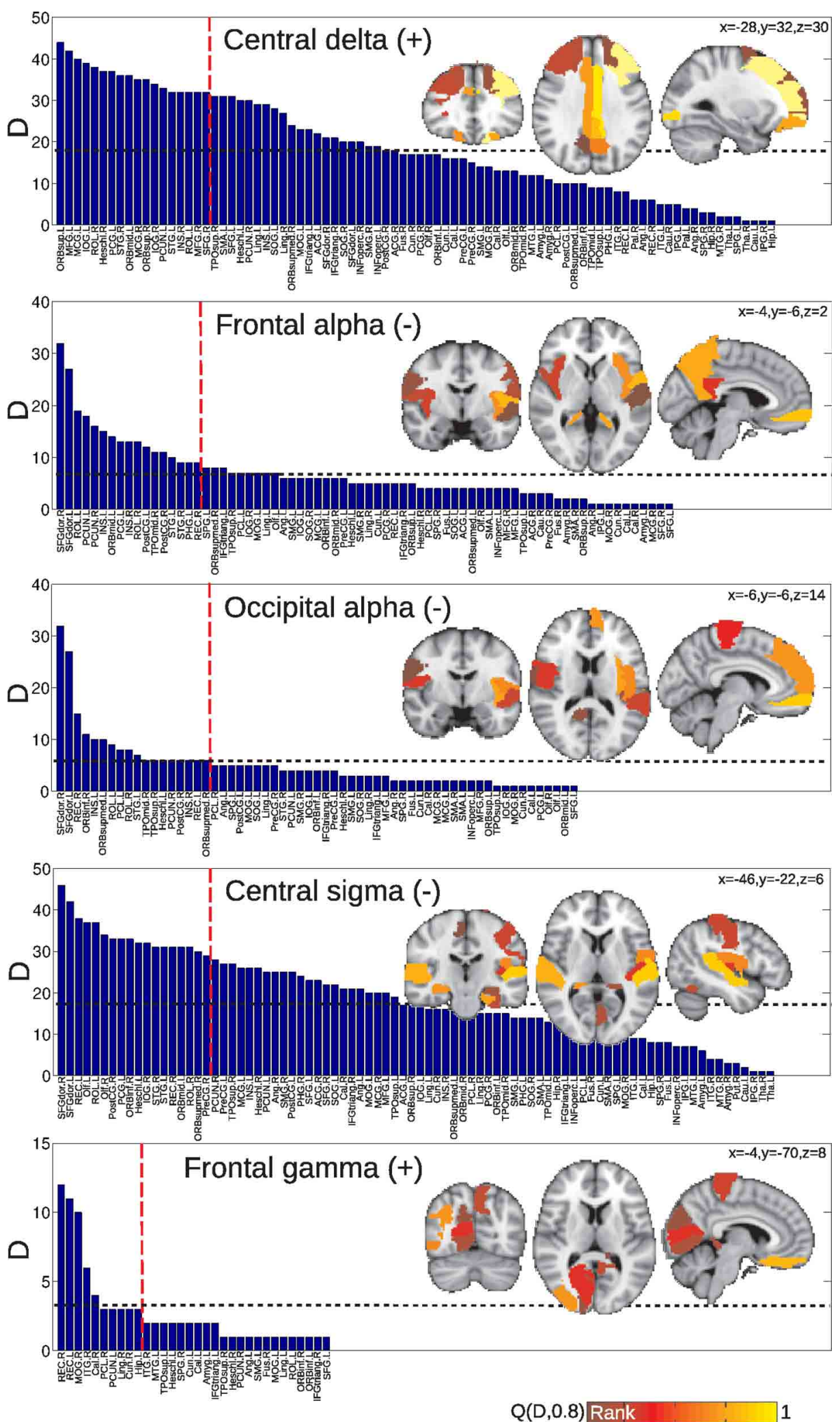

FIGURE 7 | Anatomical regions (or nodes) ranked according to the number of connections attached to them which correlate with EEG power fluctuations in different frequencies. In the inset, the regions corresponding to the top quintile of the distribution-denoted as
$Q(D, 0.8)$-are displayed overlaid on a standard MNI T1 template. The horizontal dashed line indicates the mean of the distribution. Results are for the group of subjects undergoing vigilance transitions between wakefulness and light sleep. 

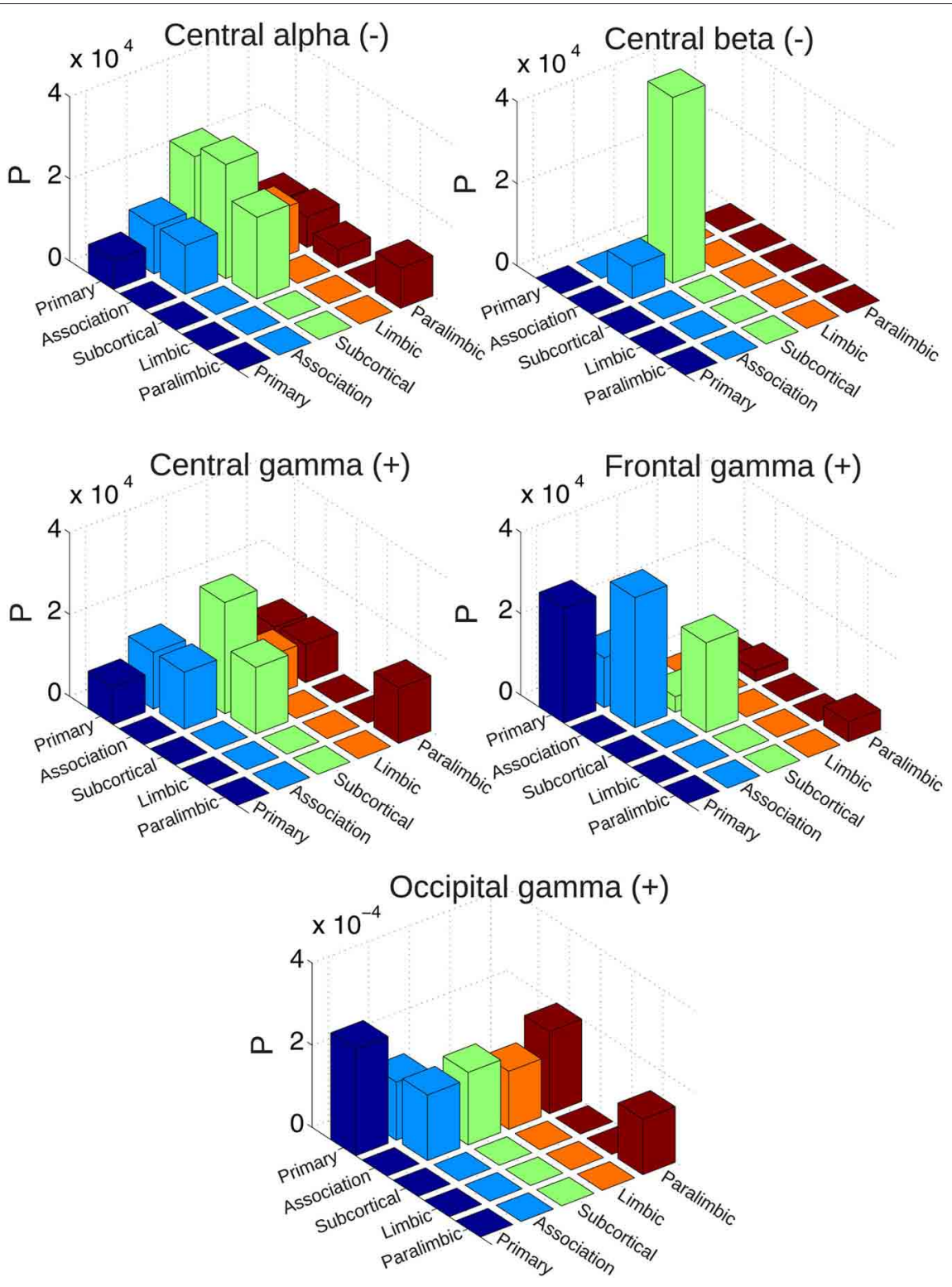

FIGURE 8 | Probability of finding connections between different systems (sensory, association, subcortical, limbic, and paralimbic) which correlate either positively or negatively with spontaneous EEG power fluctuations (normalized by the total number of possible connections between each pair of systems). Results are for the group of awake subjects. not exhibit significant correlations). These correlations were positive between average path length and frontal-central alpha. An increased average path length signals a more fragmented network, consistent with the widespread BOLD discoordination observed at times of high alpha power (Figure 4).

\section{DISCUSSION}

In this work we have studied how changes over time in BOLD functional connectivity are linked to increased local synchronization of scalp EEG rhythms, as indexed by spectral power. Our results reveal that increased EEG power in the gamma band facilitated long-range communication between brain regions (especially for those located in frontal areas), whereas frequencies in the alpha and beta range were related to diminished functional connectivity. When studied using a graph theoretical approach, these results were manifest in a positive correlation between power in the alpha band and average path length, compatible with the interpretation of less efficient connectivity between regions.

Our results are concordant with a recent study of primary visual cortex connectivity modulation by spontaneous power fluctuations in posterior alpha (Scheeringa et al., 2012), and represent an extension from regional and system-specific correlations to the global relationship between EEG frequency power changes and BOLD connectivity. Another recent study addressed the opposite question: is BOLD amplitude correlated with changes in alpha EEG phase synchronization? (Sadaghiani 

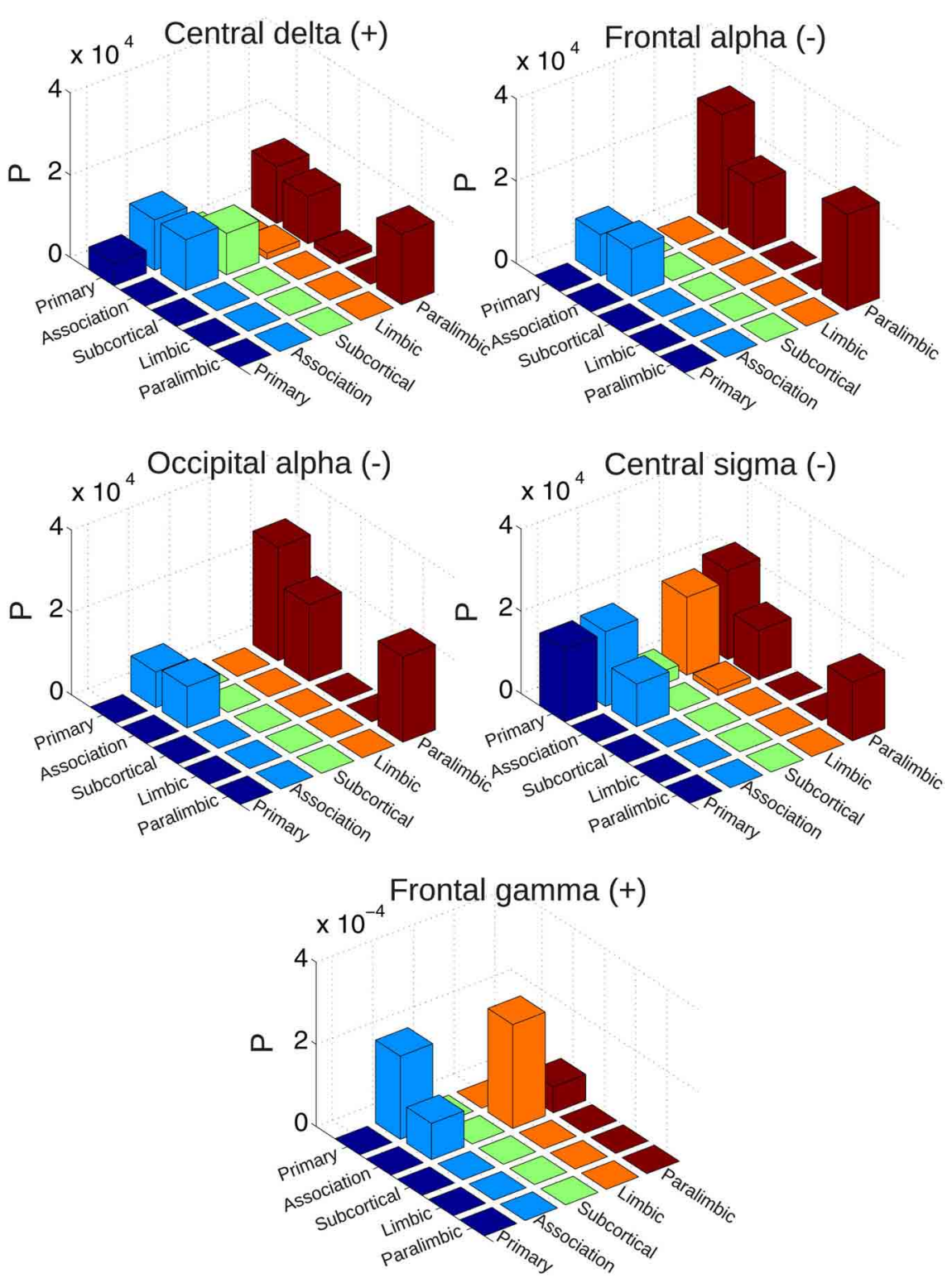

FIGURE 9 | Probability of finding connections between different systems (sensory, association, subcortical, limbic, and paralimbic) which correlate either positively or negatively with spontaneous EEG power fluctuations (normalized by the total number of possible connections between each pair of systems). Results are for the group of subjects undergoing vigilance transitions between wakefulness and light sleep. et al., 2012). Positive correlations between global synchronization and fMRI signal amplitude were found in a fronto-parietal network of regions, favoring an interpretation of alpha phase locking as related to integration of information in this cortical network. However, our direct evaluation of alpha power influence on BOLD connectivity suggests that periods of high alpha are characterized by a widespread disruption of BOLD coherence, which could be related to inhibitory process facilitating information flow in the unaffected nodes (Klimesch et al., 2007; Jensen and Mazaheri, 2010) (see discussion below).

In the following paragraphs we discuss in detail our results in the light of previous work and theories about brain function, and we develop the most important implications of our results for future studies of brain functional connectivity.

\subsection{BOLD FUNCTIONAL CONNECTIVITY FLUCTUATES AT A TIME SCALE OF MINUTES}

Our sliding window analysis revealed changes in functional connectivity at the scale of minutes. Such non-stationary connectivity can in principle be attributed to many factors or combinations of factors: intrinsic coupling and de-coupling of neural activity during rest, vigilance changes, spontaneous cognition, movement artifacts, and changes in cardiac or respiratory rates. A previous study demonstrated temporal changes in connectivity (termed 

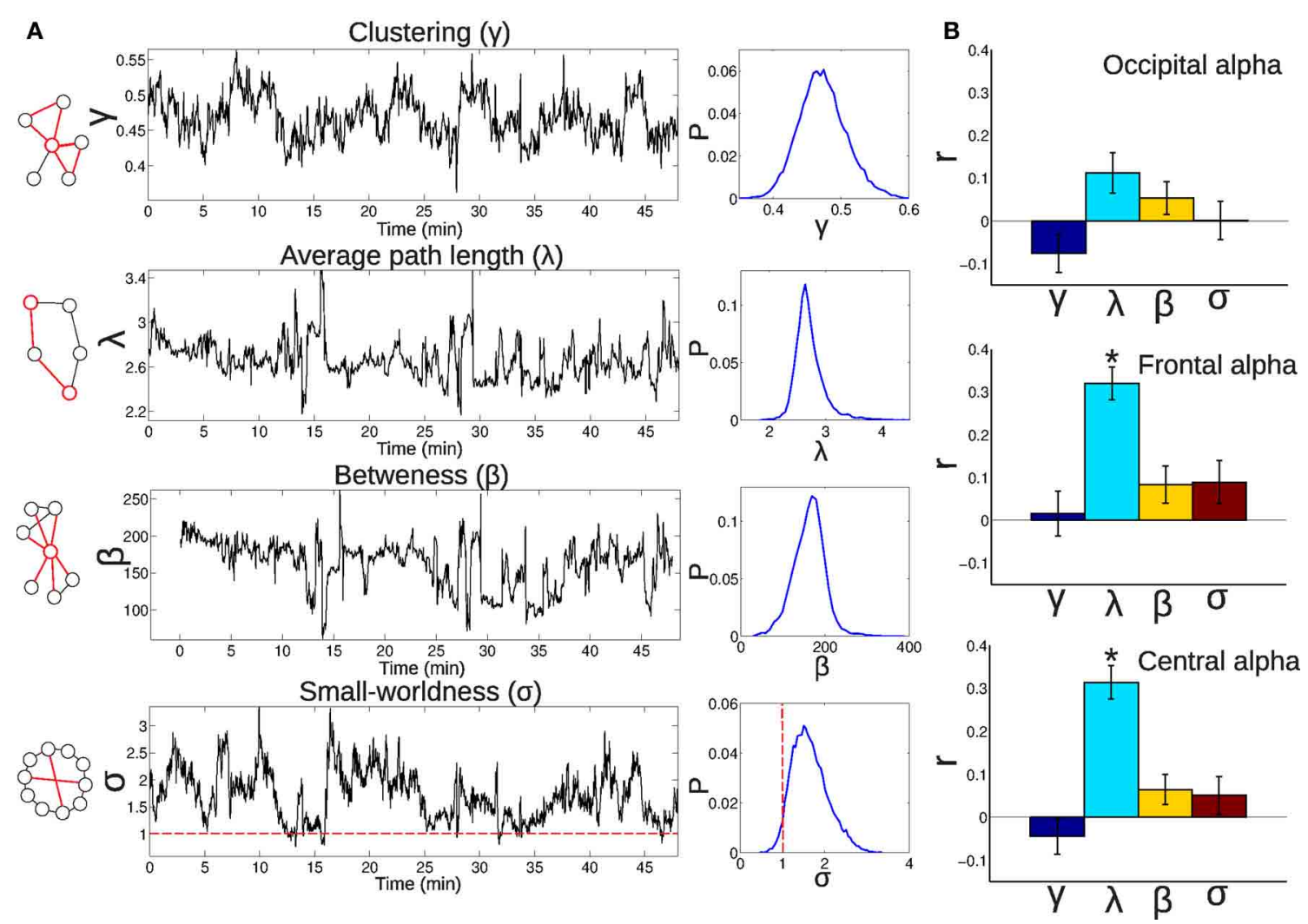

FIGURE 10 | (A) Left: Illustrations exemplifying the meaning of common graph metrics (clustering coefficient, average path length, betweeness, and small-worldness; for a detailed explanation, see the "Materials and Methods" section). Center: Examples showing the temporal evolution of the graph metrics for a single subject. Right: Probability $(P)$ distributions (for all subjects) of the graph metric values. (B) Correlations between fluctuations in the graph metrics and EEG alpha power, averaged from occipital, frontal, and central electrodes (*significant at $p<0.05$, Bonferroni corrected, $n=72$ ). dynamic functional connectivity) in anesthetized macaques while ruling out motion and spontaneous conscious cognition as the origin of the observed BOLD connectivity changes (Hutchison et al., 2012). Our study does not eliminate spontaneous cognition, rather, our EEG-fMRI analysis allows to map connectivity changes correlated with the power of scalp oscillations involved in different brain states and cognitive processes. Cardiac and motion time series did not correlate with changes in functional connectivity, and respiratory time series only correlated with a minuscule fraction of the possible connections. A large number of correlations with different EEG frequencies (discussed in detail below) - present even when motion, cardiac, and respiratory time series were taken into account as partial regressors-highlight the neural origin of the changes in BOLD connectivity: the presence of specific neural oscillations is directly related to increased or decreased temporal synchronization of BOLD signals from distinct cortical and subcortical areas.

Furthermore, the SD of sliding windowed BOLD connectivity, which quantifies the degree of deviation from a stationary connectivity, displayed structured spatial variation: connections between neighboring regions had a smaller SD, while connections between regions from heterogeneous systems showed larger deviations from constant connectivity.
Finally, our analysis included two groups of subjects: one showing steady levels of vigilance (wakefulness) and the other with transitions between wakefulness and light sleep. The onset of sleep is characterized by changes in scalp EEG oscillations [a shift from fast toward slower frequencies (AASM, 2007)]. Our analysis revealed that BOLD connectivity correlates with these spectral changes in the group of subjects falling asleep. Distributed positive temporal correlations between BOLD connectivity and EEG power in the delta band appeared, while positive correlations with the gamma band mostly disappeared. Our results also suggest an electrophysiological correlate of changes in BOLD connectivity as previously reported at sleep onset (Spoormaker et al., 2010; Tagliazucchi et al., 2012).

\subsection{NEGATIVE CORRELATIONS WITH EEG POWER FLUCTUATIONS}

We have shown that spontaneous increases in EEG alpha power (averaged over central channels) correlates with decreased BOLD connectivity between a large number of cortical and subcortical regions. This would be consistent with the proposition of alpha as an "idling rhythm" which predominates during relaxed, eyes closed rest (Pfurtscheller et al., 1996) and with that of alpha as performing functional inhibition of regions not relevant to task performance (Klimesch et al., 2007; Jensen and Mazaheri, 2010; Scheeringa et al., 2012). The BOLD connectivity decreases 
observed at times of large alpha amplitude could indicate an active suppression of sensory input and its subsequent cortical processing (Worden et al., 2000). While alpha is usually more prominent in occipital regions, the "blocking" of oscillations in the same frequency range has been related to the onset and planning of activity in sensory-motor and supplementary motor cortices (Pfurtscheller et al., 1997) [these oscillations in the alpha range are termed "mu" or "rolandic alpha rhythm" (Pfurtscheller and Andrew, 1999)]. Furthermore, previous EEG-fMRI studies demonstrated an inverse relationship between BOLD activation in a large, distributed network of cortical areas (Laufs et al., 2003a; Moosmann et al., 2003; Goncalves et al., 2006) [overlapping with the default mode network (Laufs et al., 2003b)] and EEG alpha power. Overall, these results suggest that alpha suppression is a landmark of cortical activation, a view which is expanded by the present work demonstrating a direct link with increased BOLD connectivity. Vice versa, during epochs of increased alpha oscillations, functional connectivity is-efficiently-temporally weakened.

The negative correlations obtained between BOLD connectivity and beta power are at first sight surprising, given the traditional view of these faster rhythms as a signal of increased mental activity, starting from their first observations by Berger (1938). However, desynchronization of rolandic (peri-central) beta rhythms increases cortical excitability, favoring a motor response (Deletis et al., 1992). Beta rhythms over central regions appear in synchronized fashion after (but not during) the execution of a voluntary motor command (Pfurtscheller, 1981; Pfurtscheller et al., 2003). Because of this inverse relationship between rolandic beta rhythms and cortical excitability they are, like alpha, usually considered as idling rhythms, which indicate a resting state of the sensory-motor cortex and related brain areas (Pfurtscheller and Lopez da Silva, 1999). Furthermore, an inverse relationship between peri-central beta rhythms and fMRI BOLD activation has been reported (Ritter et al., 2008). Considered together, these results suggest that both rolandic beta and alpha rhythms are related to an idling state of the cortex, which we demonstrate here related to a decreased BOLD functional connectivity. While in our study subjects did not perform any explicit motor task, we hypothesize that during rest brain activity spontaneously recapitulates activity patterns related to the execution of diverse tasks, as well as stimuli perception-a hypothesis which has received strong experimental support from different neuroimaging modalities (Ringach, 2009; Smith et al., 2009; Sadaghiani et al., 2010).

We also note that the connectivity of subcortical regions was most strongly affected by central and alpha rhythms, while primary sensory and motor cortices remained relatively unaffected. This suggests that the presence of idling rhythms in scalp EEG is related to a loss of functional connectivity between cortical areas and specific subcortical structures (e.g., thalamus for alpha rhythm and basal ganglia for beta rhythm). As an example, since spontaneous thalamic BOLD amplitude has been shown to correlate positively with EEG alpha power, and activity from a large network of cortical areas shows an inverse correlation (Laufs et al., 2003b), we can expect compromised cortico-thalamic BOLD connectivity modulations to be linked to spontaneous changes in alpha power. This is also supported by our analysis of alpha power and connectivity between different brain systems. As shown in Figure 8, the connectivity between subcortical regions and primary and association cortices [which include the regions reported in Laufs et al. (2003b)] is most compromised during periods of high alpha power. Subjects undergoing vigilance changes consistently displayed negatively correlated connectivity with alpha and sigma bands in frontal regions (bilateral superior frontal gyrus), which could be related to vigilance-related variability in correlation patterns with alpha power (Laufs et al., 2006).

\subsection{POSITIVE CORRELATIONS WITH EEg POWER FLUCTUATIONS}

In contrast to the slower frequencies, the faster gamma rhythm is almost universally related to cognitive performance. Since the first pioneering studies showing increased gamma amplitude and synchronization during visual stimulation (Gray et al., 1989), a wealth of experimental results has demonstrated the important role of activity in the gamma band during the execution of different cognitive tasks (for reviews, see Lee et al., 2003; Herrmann et al., 2004; Fries, 2005; Fries et al., 2007). The ubiquity of gamma rhythms and their apparently heterogeneous nature have led to the hypothesis that activity in the gamma band represents a fundamental process subserving an elementary operation of cortical computation (Fries, 2009). Our results closely relate increased EEG gamma power (averaged from different topographical locations) to the coordination of BOLD signals between a large number of cortical and subcortical pairs of regions. If one accepts that spontaneous fluctuations in BOLD and EEG signals resemble elicited activity patterns, this result is consistent with the experiments and hypotheses mentioned above. Such similarity is supported by studies showing that spontaneous cognitive operations underlie resting state activity fluctuations (Andrews-Hanna et al., 2010; Shirer et al., 2012). This proposition, however, cannot be held as the only origin of the aforementioned fluctuations given the coordinated spatio-temporal activity observed in states of diminished conscious awareness, such as sleep (Boly et al., 2008; Horovitz et al., 2008; Larson-Prior et al., 2009; Brodbeck et al., 2012). An alternative interpretation of BOLD signal coherence and its electrophysiological correlates during the resting state is the setting up of a baseline state favoring a quick response to environmental demands (Buckner and Vincent, 2007). In such scenario spontaneous cognition does not cause the resemblance between elicited and spontaneous activity, but the need to be close at all times to a behaviorally meaningful state.

Connectivity of frontal, precuneal and temporal regions with the rest of the brain was most strongly influenced by the increase of gamma power. EEG-fMRI studies have shown that correlations between scalp EEG gamma and BOLD activity are located predominantly in frontal regions (Mantini et al., 2007). Our study extends this observation by demonstrating a direct relationship between increased gamma and BOLD connectivity of frontal regions with the rest of the brain. Increased gamma power was related to strengthened BOLD connectivity between association, primary, and subcortical regions (Figure 8), a fact consistent with responses in the gamma band observed during cross-modal processing (Kisley and Cornwell, 2006; Yuval-Greenberg and Deouell, 2007; Senkowski et al., 2008). 
Also, we observed positive correlations between increased delta power and BOLD connectivity, but only for the subjects undergoing vigilance transitions to light sleep. The nature of scalp oscillations in the delta range is very different from those in the gamma band: their low temporal complexity reflects the alternation between neural firing ("up" state) and quiescence ("down" state). Such lack of temporal complexity, and therefore a diminished repertoire of possible neural states, was hypothesized to underlie loss of conscious awareness during sleep (Tononi et al., 1994; Tononi, 2004, 2008). Our results show that increased BOLD connectivity (suggestive of an excessive integration and a loss of functionally segregated brain modules) follows activity in the delta band for subjects transitioning to light sleep. Studies addressing the issue of temporal complexity (or temporal dependency) of BOLD signals across the human sleep cycle are needed to reveal in $\mathrm{fMRI}$ recordings the temporal properties of these slow neural oscillations.

\subsection{COMPARISON BETWEEN GROUPS}

The case for a neurophysiological origin of dynamical functional connectivity is supported by the correlations with EEG spectral power. To strengthen this observation, a manipulation can be performed in one of the variables involved in the correlation and the effect on the other can be measured. We considered the changes in EEG spectral power occurring in the transition to light sleep as such a manipulation. We indeed observed the expected changes in the correlation patterns. In contrast to the wakefulness group, the delta band was involved in strengthened BOLD connectivity, a fact expected given the general slowing of EEG frequencies which characterizes N1 sleep (see the AASM sleep scoring criteria in the "Materials and Methods" section). Increased connectivity associated with power in slow frequency bands could be related to the loss of consciousness during early sleep (see the last section of the "Discussion"). In the group of subjects undergoing transitions to light sleep, we also observed frontal and occipital alpha power to be correlated negatively with BOLD connectivity, as opposed to central alpha as in the group of awake subjects. This is expected given that the loss of alpha at sleep onset is observed predominantly in occipital channels (AASM, 2007), whereas alpha in central regions appears to be of a different nature and is associated with motor-related task demands (Pfurtscheller et al., 1997). Finally, the decreased and less widespread positive correlations with the gamma band are likely related to the loss of power in this frequency band during sleep. In particular, and as discussed in the previous section, increased BOLD connectivity of temporal, precuneal, and parietal areas during periods of high frontal gamma could relate to cross-modal binding. This effect was not observed in the group of subjects exhibiting light sleep, for whom primary sensory areas (visual) were mostly affected. A heterogeneous origin of gamma oscillations measured at these different behavioral states could underlie these differences.

\subsection{TEMPORAL SCALES AND CORRELATIONS BETWEEN BOLD CONNECTIVITY AND EEG POWER}

Given that changes in EEG power (for example, in beta or gamma bands) during or after task execution usually occur in the sub-second temporal range, it is remarkable that correlations with the connectivity of the slow, lagged, and relatively poorly sampled BOLD signal can be found. However, a period with a particularly high level of activity could elicit a change in BOLD connectivity when all the short-lived EEG power changes are considered together. This situation is analogous to that of the correlation between short periods of stable topographical configurations [EEG microstates (Koenig et al., 2002)] with specific BOLD RSN, which are likely driven by periods in which the presence of a given microstate overwhelmingly exceeds that of the others (Britz et al., 2010). It has been speculated and subsequently corroborated (Van De Ville et al., 2010) that, for this to happen, the distribution of the EEG events has to follow a scale-free distribution (or equivalently, have a $1 / f$ spectrum): only then the temporal scale invariance allows the discovery of correlations using a much slower imaging method, such as fMRI. This scale invariance is a defining property of self-organized complex systems, like the brain (Tagliazucchi and Chialvo, 2011). $1 / f$ spectra are ubiquitous in the power fluctuations of EEG, MEG, and electrocorticography (ECoG) recordings (LinkenkaerHansen et al., 2001; Miller et al., 2009; He et al., 2010; He, 2011), as well as in time series derived from cognitive and behavioral experiments (Gilden et al., 1995; Shelhamer et al., 2003).

\subsection{GRAPH METRICS FLUCTUATE OVER TIME}

We have demonstrated that during the time evolution of wholebrain functional networks, associated graph metrics (clustering coefficient, average path length, betweeness, and smallworldness) also change over time. Given this result, a number of recent studies based on the methodology of graph theory have to be re-interpreted. The reported value of the different graph metrics cannot be taken as a constant property of brain networks, instead, it has to be considered as an asymptotic property (i.e., the value one obtains during a long measurement) emerging after temporal averaging. There are two interesting immediate consequences of this observation. First, resting state studies applying graph theoretical methods should be based on long recordings, since short acquisition times will decrease the confidence on the graph metric estimates (the risk of computing them in a period in which they largely deviate from the mean will be higher). Second, the temporal evolution of graph metrics should be taken into account. For example, when comparing two populations using graph theoretical methods, equal values of the associated graph metrics may be obtained, yet their dynamical behavior could be completely different. Further investigations are needed to study this and other possibilities of considering the evolution of functional networks over time.

\subsection{IMPLICATIONS FOR THEORIES OF CONSCIOUS BRAIN FUNCTION}

Our results show that the onset of specific (fast) rhythms is accompanied by distributed binding of BOLD activity (i.e., increased functional connectivity), whereas other (slower) oscillations rather inhibit such binding, decreasing the overall corticocortical and cortico-subcortical connectivity. The fact that long-range functional connectivity in the human brain is unstable 
and fluctuates in a coordinated fashion with fast EEG rhythms likely reflects the dynamic nature of processes underlying the conscious state. For instance, an approach to consciousness focusing in its nature as a process (instead of a state or a capacity) emphasizes the presence of a dynamical core, a continuously changing set of neuronal groups strongly integrated together during hundreds of milliseconds and allowing differentiated responses, i.e., having a large neural complexity (Tononi and Edelman, 1998). Such complexity is hindered in cases of overly integrated or segregated dynamics, such as those present during sleep (Tononi et al., 1994; Tononi, 2004, 2008). In this framework, the higher large-scale BOLD functional connectivity associated with increased delta power could be related to the loss of consciousness which occurs during early sleep. Interestingly, an opposite scenario (increased functional segregation) was reported in a recent study (Boly et al., 2012). This seeming discrepancy could arise because all NREM sleep stages were considered together (including N2 and N3, indicating also deeper sleep).

While dynamical functional connectivity and its electrophysiological characteristics are suggestive of the processes postulated by the aforementioned theories, further experimental tests are required in order to corroborate them as a correlate of conscious awareness (for example, studying whether these interrelated, dynamical landmarks of brain activity are also prevalent during deeper sleep stages, anesthesia or coma).

\section{REFERENCES}

AASM. (2007). The AASM Manual for the Scoring of Sleep and Associated Events-Rules, Terminology and Technical Specifications. Chicago: American Academy of Sleep Medicine.

Achard, S., Salvador, R., Whitcher, B., Suckling, J., and Bullmore, E. (2006). A resilient, low-frequency, small-world human brain functional network with highly connected association cortical hubs. J. Neurosci. 26, 63-72.

Allen, P. J., Polizzi, G., Krakow, K., Fish, D. R., and Lemieux, L. (1998). Identification of EEG events in the MR scanner: the problem of pulse artifact and a method for its subtraction. Neuroimage 8, 229-239.

Andrews-Hanna, J. R., Reidler, J. S., Huang, C., and Buckner, R. L. (2010). Evidence for the default network's role in spontaneous cognition. J. Neurophysiol. 104, 322-335.

Bassett, D. S., Wymbs, N. F., Porter, M. A., Mucha, P. J., Carlson, J. M., and Grafton, S. T. (2010). Dynamic reconfiguration of human brain networks during learning. Proc. Natl. Acad. Sci. U.S.A. 108, 7641-7646.

Berger, H. (1938). Über das Elektroenkephalogram des Menschen. Vierzehnte Mitteilung.
Arch. Psychiat. Nervenkr. 108, 407-431.

Boly, M., Phillips, C., Tshibanda, L., Vanhaudenhuyse, A., Schabus, M., Dang-Vu, T. T., et al. (2008). Intrinsic brain activity in altered states of consciousness. How conscious is the default mode of brain function? Ann. N.Y. Acad. Sci. 1129, 119-129.

Boly, M., Perlbarg, V., Marrelec, G., Schabus, M., Laureys, S., Doyon, J., et al. (2012). Hierarchical clustering of brain activity during human nonrapid eye movement sleep. Proc. Natl. Acad. Sci. U.S.A. 109, 5856-5861.

Britz, J., van de Ville, D., and Michel, C. M. (2010). BOLD correlates of EEG topography reveal rapid resting-state network dynamics. Neuroimage 52, 1162-1170.

Brodbeck, V., Kuhn, A., von Wegner, F., Morzelewski, A., Tagliazucchi, E., Borisov, S., et al. (2012). EEG microstates of wakefulness and NREM sleep. Neuroimage 62, 2129-2139.

Buckner, R., and Vincent, J. (2007). Unrest at rest: default activity and spontaneous network correlations. Neuroimage 37, 1091-1096.

Bullmore, E., and Sporns, O. (2009). Complex brain networks: graph theoretical analysis of structural and functional systems. Nat. Rev. Neurosci. 10, 186-198.

\subsection{CONCLUSION}

Large efforts have been devoted to the identification of the electrophysiological correlates of the fMRI BOLD contrast. We have approached this problem from a new perspective: studying whether band-specific scalp oscillations are related to increased (or decreased) BOLD coherence instead of directly relating them to changes in BOLD amplitude. This approach has allowed us to observe, for the first time, a relationship between BOLD signal functional connectivity and increased local synchronization in the gamma band. Furthermore, slower "idling" rhythms were linked to large-scale disconnection patterns, also quantified by correlation with adequate graph metrics. The relationship found between EEG power fluctuations and dynamic BOLD functional connectivity leads us to conclude that this phenomenon is very likely to be of neuronal origin, and thus it deserves even further investigation.

\section{ACKNOWLEDGMENTS}

This work was funded by the Bundesministerium für Bildung und Forschung (grant 01 EV 0703) and the LOEWE Neuronale Koordination Forschungsschwerpunkt Frankfurt (NeFF). The authors thank Torben E. Lund for providing a MATLAB implementation of the RETROICOR method, Sandra Anti, Ralf Deichmann, and Steffen Volz for extensive MRI support, and all subjects for their participation.

Bullmore, E., and Sporns, O. (2012). The economy of brain network organization. Nat. Rev. Neurosci. 13, 336-349

Buzsáki, G. (2006). Rhythms of the Brain. New York, NY: Oxford University Press.

Chang, C., and Glover, G. H. (2010). Time-frequency dynamics of resting-state brain connectivity measured with fMRI. Neuroimage 50, 81-98.

Deletis, V., Schild, J. H., Beric, A., and Dimitrijevic, M. R. (1992). Facilitation of motor evoked potentials by somatosensory afferent stimulation. Electroencephalogr. Clin. Neurophysiol. 85, 302-310.

Donner, T. H., Siegel, M., Fries, P., and Engel, A. K. (2009). Buildup of choice-predictive activity in human motor cortex during perceptual decision making. Curr. Biol. 19, 1581-1585.

Fraiman, D., and Chialvo, D. R. (2012). What kind of noise is brain noise: anomalous scaling behavior of the resting brain activity fluctuations. Front. Physio. 3:307. doi: 10.3389/fphys.2012.00307

Fries, P. (2005). A mechanism for cognitive dynamics: neuronal communication through neuronal coherence. Trends Cogn. Neurosci. 9, 474-480.

Fries, P. (2009). Neuronal gamma-band synchronization as a fundamental process in cortical computation. Annu. Rev. Neurosci. 32, 209-224.

Fries, P., Nikolic D., and Singer W. (2007). The gamma cycle. Trends Neurosci. 30, 309-316

Glover, G. H., Li, T. Q., and Ress, D. (2000). Image-based method for retrospective correction of physiological motion effects in fMRI: RETROICOR. Magn. Reson. Med. 44, 162-167.

Goncalves, S. I., de Munck, J. C., Pouwels, P. J. W., Schoonhoven, R., Kuijer, J. P. A., Maurits, N. M., et al. (2006). Correlating the alpha rhythm to BOLD using simultaneous EEG/fMRI: inter-subject variability. Neuroimage 30, 203-213.

Gilden, D. L., Thornton, T., and Mallon, M. W. (1995). 1/f noise in human cognition. Science 267, 1837-1839.

Gray, C. M., König, P., Engel, A. K., and Singer, W. (1989). Oscillatory responses in cat visual cortex exhibit inter-columnar synchronization which reflects global stimulus properties. Nature 338, 334-338.

Handwerker, D. A., Roopchansingh, V., Gonzalez-Castillo, J., and Bandettini, P. A. (2012). Periodic changes in fMRI connectivity. Neuroimage 63, 1712-1719.

He, B. J. (2011). Scale-Free properties of the functional magnetic resonance imaging signal during rest and task. J. Neurosci. 31, 13786-13795. 
He, B. J., Zempel, J. M., Snyder, A. Z., and Raichle, M. E. (2010). The temporal structures and functional significance of scale-free brain activity. Neuron 66, 353-369.

Herrmann, C. S., Munk, M. H. J., and Engel, A. K. (2004). Cognitive functions of gamma-band activity: memory match and utilization. Trends Cogn. Neurosci. 8, 347-355.

Horovitz, S. G., Fukunaga, M., de Zwart, J. A., van Gelderen, P., Fulton, S. C., Balkin, T. J., et al. (2008). Low frequency BOLD fluctuations during resting wakefulness and light sleep: a simultaneous EEG-fMRI study. Hum. Brain Mapp. 29, 671-682.

Humphries, M. D., Gurney, K., and Prescott, T. J. (2006). The brainstem reticular formation is a small-world, not scale-free, network. Proc. Biol. Sci. 273, 503-511.

Hutchison, R. M., Womelsdorf, T., Gati, J. S., Everling, S., and Menon, R. S. (2012). Resting-state networks show dynamic functional connectivity in awake humans and anesthetized macaques. Hum. Brain Mapp. doi: 10.1002/hbm.22058. [Epub ahead of print].

Jensen, O., and Mazaheri, A. (2010). Shaping functional architecture by oscillatory alpha activity: gating by inhibition. Front. Hum. Neurosci. 4:186. doi: 10.3389/fnhum.2010.00186

Kisley, M. A., and Cornwell, Z. M. (2006). Gamma and beta neural activity evoked during a sensory gating paradigm: effects of auditory, somatosensory and crossmodal stimulation. Clin. Neurophys. 117, 2549-2563.

Klimesch W., Sauseng P., and Hanslmayr S. (2007). EEG alpha oscillations: the inhibition-timing hypothesis. Brain Res. Rev. 53, 63-88.

Koenig, T., Prichep, L., Lehmann D., Valdes Sosa, P., Braeker, E., Kleinlogel, H., et al. (2002). Millisecond by millisecond, year by year: normative EEG microstates and developmental stages. Neuroimage 16, 41-48.

Larson-Prior, L. J., Zempel, J. M., Nolan, T. S., Prior, F. W., Snyder, A. Z., and Raichle, M. E. (2009). Cortical network functional connectivity in the descent to sleep. Proc. Natl. Acad. Sci. U.S.A. 106, 4489-4494.

Laufs, H., Holt, J. L., Elfont, R., Krams, M., Paul, J. S., Krakow, K., et al. (2006). Where the BOLD signal goes when alpha EEG leaves. Neuroimage 31, 1408-1418.
Laufs, H., Kleinschmidt, A., Beyerle, A., Eger, E., Salek-Haddadi, A., Preibisch, C., et al. (2003a). EEGcorrelated fMRI of human alpha activity. Neuroimage 19, 1463-1476.

Laufs, H., Krakow, K., Sterzer, P., Eger, E., Beyerle, A., SalekHaddadi, A., et al. (2003b). Electroencephalographic signatures of attentional and cognitive default modes in spontaneous brain activity fluctuations at rest. Proc. Natl. Acad. Sci. U.S.A. 100, 11053-11058.

Lee, K. H., Williams, L. M., Breakspear, M., and Gordon, E. (2003). Synchronous gamma activity: a review and contribution to an integrative neuroscience model of schizophrenia. Brain Res. Rev. 41, 57-78.

Linkenkaer-Hansen, K., Nikouline, V. V., Palva, J. M., and Ilmoniemi, R. J. (2001). Long-range temporal correlations and scaling behavior in human brain oscillations. J. Neurosci. 21, 1370-1377.

Logothetis, N. K., Pauls, J., Augath, M., Trinath, T., and Oeltermann, A. (2001). Neurophysiological investigation of the basis of the fMRI signal. Nature 412, 150-157.

Logothetis, N. K., and Pfeuffer, J. (2004). On the nature of the BOLDf-MRI contrast mechanism. Magn. Reson. Imag. 22, 1517-1531.

Mantini, D., Perrucci, M. G., Del Gratta, C., Romani, G. L., and Corbetta, M. (2007). Electrophysiological signatures of resting state networks in the human brain. Proc. Natl. Acad. Sci. U.S.A. 104, 13170-13175.

Melloni, L., Molina, C., Pena, M., Torres, D., Singer, W., and Rodriguez E. (2007). Synchronization of neural activity across cortical areas correlates with conscious perception. J. Neurosci. 27, 2858-2865.

Miller, K. J., Sorensen, L. B., Ojemann, J. G., and den Nijs, M. (2009). Power-law scaling in the brain surface electric potential. PLoS Comput. Biol. 5:e1000609. doi: 10.1371/journal.pcbi.1000609

Moosmann, M., Ritter, P., Krastel, I., Brink, A., Thees, S., Blankenburg, F., et al. (2003). Correlates of alpha rhythm in functional magnetic resonance imaging and near infrared spectroscopy. Neuroimage 20, 145-158.

Petridou, N., Caballero Gaudes, C., Dryden, I. L., Francis, S. T., and Gowland, P. A. (2012). Periods of rest in fMRI contain individual spontaneous events which are related to slowly fluctuating spontaneous activity. Hum. Brain Mapp. doi: 10.1002/hbm.21513. [Epub ahead of print].

Pfurtscheller, G. (1981). Central beta rhythm during sensorimotor activities in man. Electroencephalogr. Clin. Neurophysiol. 51, 253-264.

Pfurtscheller, G., and Andrew, C. (1999). Event-related changes of band power and coherence: methodology and interpretation. $J$. Clin. Neurophysiol. 16, 512-519.

Pfurtscheller, G., Graimann, B. Huggins, J. E., Levine, S. P., and Schuh, L. A. (2003). Spatiotemporal patterns of beta desynchronization and gamma synchronization in corticographic data during self-paced movement. Clin. Neurophysiol. 114 1226-1236.

Pfurtscheller, G., and Lopez da Silva, F. H. (1999). Event-related EEG/MEG synchronization and desyhncronization: basic principles. Clin. Neurophys. 110, 1842-1857.

Pfurtscheller, G., Neuper, C., Flotzinger D., and Pregenzer M. (1997). EEGbased discrimination between imagination of right and left hand movement. Electroencephalogr. Clin. Neurophysiol. 103, 642-651.

Pfurtscheller, G., Stancak, A., and Neuper, C. (1996). Event-related synchronization (ERS) in the alpha band- an electrophysiological correlate of cortical idling: a review. Int. J. Psychophysiol. 24, 39-46.

Raichle, M. E., MacLeod, A. M., Snyder, A. Z., Powers, W. J., Gusnard, D. A., and Shulman, G. L. (2001). A default mode of brain function. Proc. Natl. Acad. Sci. U.S.A. 98, 676-682.

Ringach, D. L. (2009). Spontaneous and driven cortical activity: implications for computation. Curr. Opin. Neurobiol. 19, 439-444.

Ritter, P., Moosmann, M., and Villringer, A. (2008). Rolandic alpha and beta EEG rhythms' strengths are inversely related to fMRI-BOLD signal in primary somatosensory and motor cortex. Hum. Brain Mapp. 30, 1168-1187.

Rubinov, M., and Sporns, O. (2010). Complex network measures of brain connectivity: uses and interpretations. Neuroimage 52, 1059-1069.

Sadaghiani, S., Hesselmann, G., Friston, K. J., and Kleinschmidt, A. (2010). The relation of ongoing brain activity, evoked neural responses, and cognition. Front. Syst. Neurosci. 4:20. doi: 10.3389/fnsys.2010.00020

Sadaghiani, S., Scheeringa, R., Lehongre, K., Morillon, B., Giraud, A. L., D'Esposito, M., et al. (2012). Alpha-band phase synchrony is related to activity in the frontoparietal adaptive control network. $J$ Neurosci. 32, 14305-14310.

Scheeringa, R., Petersson, K. M. Kleinschmidt, A., Jensen, O., and Bastiaansen, M. C. (2012). EEG alpha power modulation of fMRI resting state connectivity. Brain Connect. 2, 254-264.

Schölvinck, M. K., Maier, A., Ye, F. Q., Duyn, J. H., and Leopold, D. A. (2010). Neural basis of global resting-state fMRI activity. Proc. Natl. Acad. Sci. U.S.A. 107, 10238-10243.

Senkowski, D., Schneider, T. R., Foxe, J. J., and Engel, A. K. (2008). Crossmodal binding through neural coherence: implications for multisensory processing. Trends Neurosci. 31, 401-409.

Shelhamer, M., and Joiner, W. M. (2003). Saccades exhibit abrupt transition between reactive and predictive, predictive saccade sequences have long-term correlations. J. Neurophys. 90, 2763-2769.

Shirer, W. R., Ryali, S., Rykhlevskaia, E., Menon, V., and Greicius, M. D. (2012). Decoding subject-driven cognitive States with whole-brain connectivity patterns. Cereb. Cortex 22, 158-165.

Singer, W., Engel, A. K., Kreiter A. K., Munk M. H. J., Neuenschwander S., and Roelfsema P. R. (1997). Neuronal assemblies: necessity, signature and detectability. Trends Cogn. Sci. 1, 252-261.

Smith, S. M., Fox, P. T., Miller, K. L., Glahn, D. C., Fox, P. M., MacKay, C. E., et al. (2009). Correspondence of the brain's functional architecture during activation and rest. Proc. Natl. Acad. Sci. U.S.A. 106, 13040-13045.

Smith, S. M., Miller, K. L., Moeller, S., $\mathrm{Xu}$, J., Auerbach, E. J., Woolrich, M. W., et al. (2012). Temporallyindependent functional modes of spontaneous brain activity. Proc. Natl. Acad. Sci. U.S.A. 109, 3131-3136.

Smith, S. M., Miller, K. L., SalimiKhorshidi, G., Webster, M., Beckmann, C. F., Nichols, T. E., et al. (2011). Network modelling methods for FMRI. Neuroimage 54, 875-891.

Spoormaker, V. I., Schröter M., Gleiser, P. M., Andrade, K. C., Dresler, M., Wehrle, R., et al. (2010). Development of a large-scale functional brain network during human non-rapid eye movement sleep. J. Neurosci. 20, 11379-11387.

Sporns, O., Chialvo, D. R., Kaiser, M., and Hilgetag, C. C. (2004). Organization, development and 
function of complex brain networks. Trends. Cogn. Neurosci. 8, 418-425.

Tagliazucchi, E., Balenzuela, P., Fraiman, D., and Chialvo, D. R. (2010a). Brain resting state is disrupted in chronic back pain patients. Neurosci. Lett. 485, 26-31.

Tagliazucchi, E., Balenzuela, P., Fraiman, D., Montoya, P., and Chialvo, D. R. (2010b). Spontaneous BOLD event triggered averages for estimating functional connectivity at resting state. Neurosci. Lett. 488, 158-163.

Tagliazucchi, E., Balenzuela, P., Fraiman, D., and Chialvo, D. R. (2012). Criticality in large-scale brain fMRI dynamics unveiled by a novel point process analysis. Front. Physio. 3:15. doi: 10.3389/fphys.2012.00015.

Tagliazucchi, E., and Chialvo, D. R. (2011). "The collective brain," in Decision Making: A Psychophysics Application of Network Science (center for nonlinear science, University of North Texas, USA), 10-13.

Tagliazucchi, E., von Wegner, F., Morzelewski, A., Borisov, S., Jahnke, K., and Laufs, H. (2012). Automatic sleep staging using
fMRI functional connectivity data. Neuroimage 63, 63-72.

Tononi, G. (2004). An information integration theory of consciousness. BMC Neurosci. 5:42. doi: 10.1186/1471-2202-5-42

Tononi, G. (2008). Consciousness as integrated information: a provisional manifesto. Biol. Bull. 215, 216-242.

Tononi, G., and Edelman, G. M. (1998). Consciousness and complexity. Science 282, 1846-1851.

Tononi, G., Sporns, O., and Edelman, G. M. (1994). A measure for brain complexity: relating functional segregation and integration in the nervous system. Proc. Natl. Acad. Sci. U.S.A. 91, 5033-5037.

Tzourio-Mazoyer, N., Landeau, B., Papathanassiou, D., Crivello, F., Etard, O., Delcroix, N., et al. (2002). Automated anatomical labeling of activations in SPM using a macroscopic anatomical parcellation of the MNI MRI singlesubject brain. Neuroimage 15, 273-289.

Van De Ville, D., Britz, J., and Michel, C. M. (2010). EEG microstate sequences in healthy humans at rest reveal scale-free dynamics.
Proc. Natl. Acad. Sci. U.S.A. 107, 18179-18184.

van den Heuvel, M. P., Stam, C. J., Boersma, M., and Hulshoff Pol, $\mathrm{H}$ E. (2008). Small-world and scalefree organization of voxel-based resting-state functional connectivity in the human brain. Neuroimage 43 , 528-539.

Van Dijk, K. R. A., Hedden, T., Venkataraman, A., Evans, K. C., Lazar, S. W., and Buckner, R. L. (2010). Intrinsic functional connectivity as a tool for human connectomics: theory, properties, and optimization. J. Neurophys. 103, 297-321.

Womelsdorf, T., and Fries, P. (2007) The role of neuronal synchronization in selective attention. Curr. Opin. Neurobiol. 17, 54-160.

Worden, M. S., Foxe, J. J., Wang, N., and Simpson, G. V. (2000) Anticipatory biasing of visuospatial attention indexed by retinotopically specific $\alpha$-band electroencephalography increases over occipital cortex. J. Neurosci. 20:RC63.

Yuval-Greenberg, S., and Deouell, L. Y. (2007). What you see is not (always) what you hear: induced gamma band responses reflect cross-modal interactions in familiar object recognition. J. Neurosci. 27, 1090-1096.

Conflict of Interest Statement: The authors declare that the research was conducted in the absence of any commercial or financial relationships that could be construed as a potential conflict of interest.

Received: 06 October 2012; accepted: 09 December 2012; published online: 28 December 2012.

Citation: Tagliazucchi E, von Wegner $F$, Morzelewski A, Brodbeck $V$ and Laufs $H$ (2012) Dynamic BOLD functional connectivity in humans and its electrophysiological correlates. Front. Hum. Neurosci. 6:339. doi: 10.3389/fnhum. 2012.00339

Copyright (c) 2012 Tagliazucchi, von Wegner, Morzelewski, Brodbeck and Laufs. This is an open-access article distributed under the terms of the Creative Commons Attribution License, which permits use, distribution and reproduction in other forums, provided the original authors and source are credited and subject to any copyright notices concerning any third-party graphics etc. 


\section{APPENDIX}

In Figures $\mathbf{A} \mathbf{1}$ and $\mathbf{A} \mathbf{2}$ all the mean correlation matrices (for both groups, all frequencies and scalp locations) are reproduced for comparison purposes. Those matrices including significant correlations (also reproduced in Figures $\mathbf{4}$ and $\mathbf{5}$ ) are marked with an asterisk.

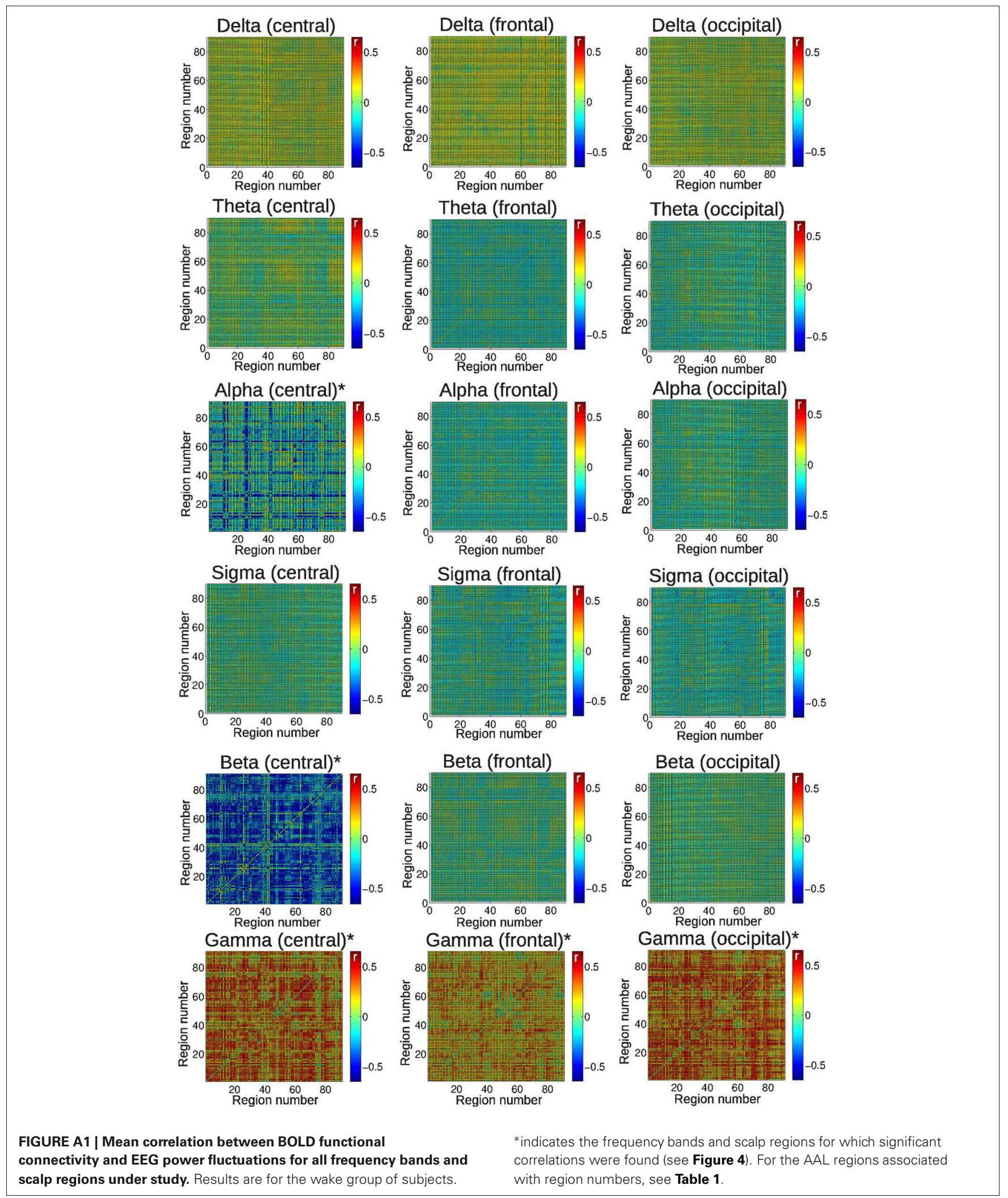




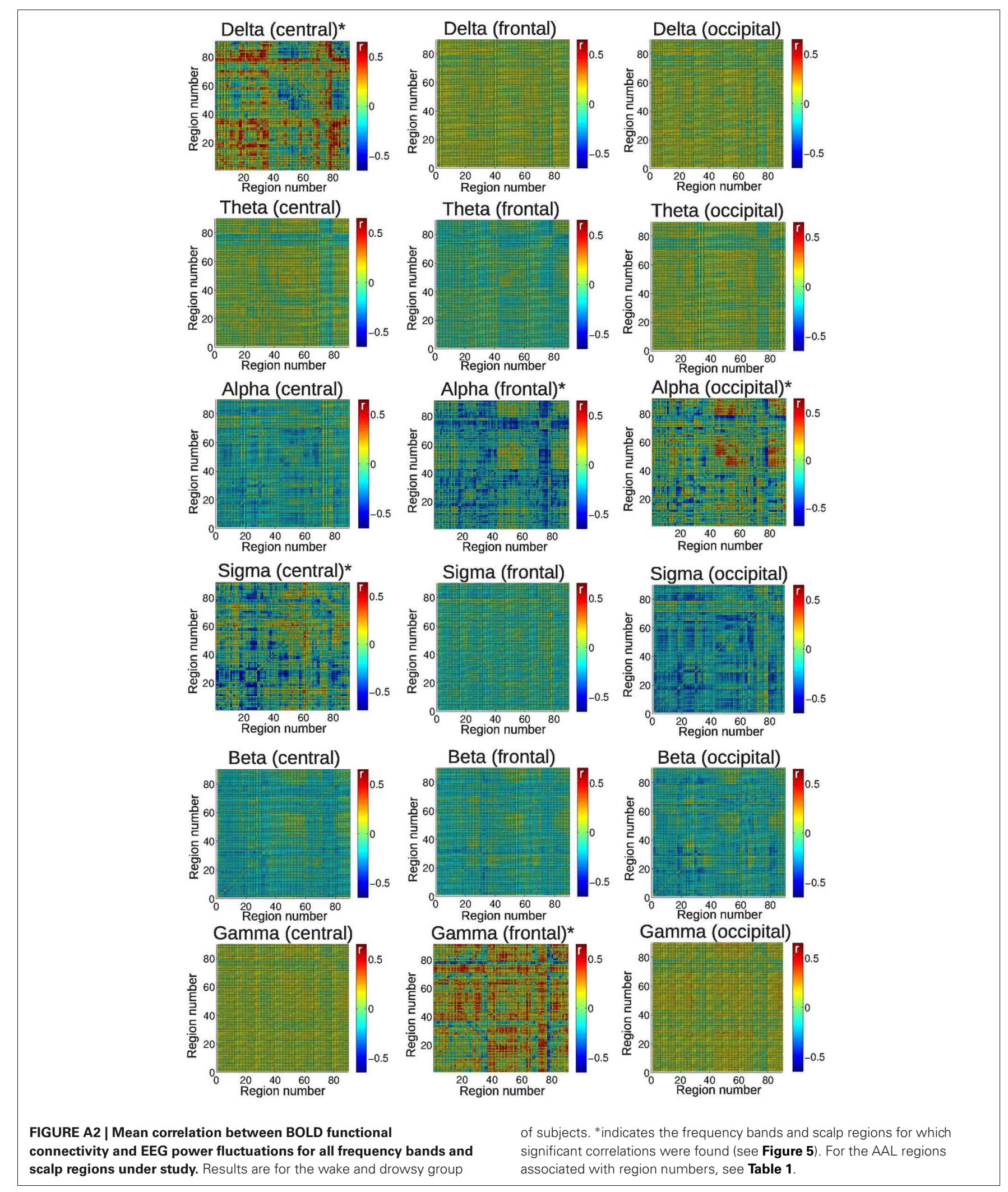

